\title{
Robustness Analysis of Nonlinear Observers for the Slow Variables of Singularly Perturbed Systems
}

\author{
Luis Cuevas* | Dragan Nešić | Chris Manzie
}

Department of Electrical and Electronic Engineering, The University of Melbourne, Melbourne, Australia

\section{Correspondence}

*Luis Cuevas, Department of Electrical and Electronic Engineering, The University of Melbourne, Parkville VIC 3010, Australia.

Email:1cuevas@student.unimelb.edu.au

\section{Present Address}

The University of Melbourne, Parkville VIC 3010, Australia.

This work was supported by the Melbourne International Research Scholarship scheme of The University of Melbourne, and by the Australian Research Council Discovery Project (DP170104102).

\begin{abstract}
Summary
Estimation of unmeasured variables is a crucial objective in a broad range of applications. However, the estimation process turns into a challenging problem when the underlying model is nonlinear and even more so when additionally it exhibits multiple time scales. The existing results on estimation for systems with two-time scales apply to a limited class of nonlinear plants and observers. We focus on analysing nonlinear observers designed for the slow state variables of nonlinear singularly perturbed systems. Moreover, we consider the presence of bounded measurement noise in the system. We generalise current results by considering broader classes of plants and estimators to cover reduced-order, full-order and higher-order observers. First, we show that the singularly perturbed system has bounded solutions under an appropriate set of assumptions on the corresponding boundary layer and reduced systems. We then exploit this property to prove that, under reasonable assumptions, the error dynamics of the observer designed for the reduced system are semi-globally inputto-state (ISS) practically stable when the observer is implemented on the original plant. We also conclude $\mathcal{L}_{2}$ stability results when the measurement noise belongs to $\mathcal{L}_{2} \cap \mathcal{L}_{\infty}$. In the absence of measurement noise, we state results on semi-global practical asymptotical (SPA) stability for the error dynamics. We illustrate the generality of our main results through three classes of systems with corresponding observers and one numerical example.
\end{abstract}

\section{KEYWORDS:}

Singularly Perturbed Systems, Observer Design, Semi-global Practical Asymptotical Stability, Input-toState Stability, $\mathcal{L}_{2}$ Stability.

\section{1 | INTRODUCTION}

In general, it is infeasible or prohibitively expensive to measure all variables of interest in a dynamic system. When state variables are required and not measured, they need to be estimated using observers. There exists a robust estimation framework for linear plant models based on the Luenberger observer and Kalman filter. These linear estimation techniques have been applied to nonlinear systems after linearisation of the system dynamics. Nevertheless, those observers are valid only locally and only work well when the system is evolving close to the equilibrium point considered for the linearisation. To counteract this problem, a wide variety of nonlinear observer design methods have been developed ${ }^{1+10}$, but these approaches may lead to ill-conditioned gains when used for systems exhibiting multiple time scales. 
Although systems with multiple time scales are standard in a wide range of applications, observers for general nonlinear systems exhibiting time-scale separation are missing. Power electronic systems $\frac{11}{11}$, chemical process ${ }^{12 \mid 13}$, electrochemical systems ${ }^{14}$, biological processes ${ }^{11}$ and electromechanical systems ${ }^{15}$ are just some examples in which multiple time-scales arise. Observer design for linear singularly perturbed systems has been studied previously 16 .19. As far as we are aware, there are limited results on nonlinear estimation for systems with two-time scales. For instance, an approach for the estimation of slow variables of a deterministic singularly perturbed system is presented in Kazantzis et al ${ }^{1]}$. The authors in Kazantzis et al ${ }^{1]}$ only consider a particular class of plants and a specific nonlinear observer that exhibits linear error dynamics for the slow system. An observer for spring-mass-damper systems with singularly perturbed structure is given in Saha and Valasek ${ }^{617}$; however, those results apply to a very specific class of systems and observers. Other results on observer design for nonlinear singularly perturbed systems are available in Wang and Liu ${ }^{20}$, and Darrogheh et al 21 .

We recently reported results on SPA stability for a general class of plants and full-order observers in Cuevas et al ${ }^{22}$. We illustrated and demonstrated the applicability of those results in Cuevas et al ${ }^{23}$. Moreover, we presented global results to cover globally Lipschitz nonlinear systems in Cuevas et $\mathrm{al}^{24}$. To generalise findings in Kazantzis et al ${ }^{11}$ and Cuevas et al ${ }^{22 \mid}{ }^{24}$, here we consider a broader class of systems and nonlinear observers of general dimension. Furthermore, we deal with the case where the measured output is corrupted by measurement noise. Here, we call measurement noise to a external disturbance with appropriately bounded amplitude, which can be high or low frequency. The stochastic properties of the high-frequency noise are outside of the scope of this manuscript. Our goal is to analyse the robustness with respect to singular perturbations and measurement noise of nonlinear observers designed to estimate the slow states of a nonlinear singularly perturbed system when the input and output are available. We do not consider the fast variables for the observer design process; instead, the observer is designed on the basis of the reduced (slow) model. We work with this approach since model-based observer design for singularly perturbed systems may lead to ill-conditioned observer gains, and subsequently, to undesired convergence properties of the estimation error if the observer is designed for the full plant. An important case where our results can be applied is the class of systems in which the sensor and/or actuator dynamics are much faster than the dynamics of the plant. The fast (sensor/actuator) states are typically not needed in such situations; hence, we need to estimate only the slow variables of the process.

We provide a general estimation framework for nonlinear singularly perturbed systems in the standard form. To the best of our knowledge, there are no known general results in the form we state ours. By taking advantage of the cascade properties of the observer and error dynamics, we prove that, under general conditions on observer and plant, the estimation error is semi-globally input-to-state practically stable where the slow and fast states are seen as inputs. Moreover, we show that the ISS property leads to SPA stability in the singular perturbation parameter $\epsilon$. We also provide $\mathcal{L}_{2} \cap \mathcal{L}_{\infty}$ stability results to cover cases where the noise is a bounded input signal that belongs to $\mathcal{L}_{2} \cap \mathcal{L}_{\infty}$. To analyse the observer design problem, we first show that, under certain assumptions on the reduced and boundary layer systems, the original system exhibits an input-to-state practical stability property with respect to the input and its derivative (practical DISS) as well as a practical $\mathcal{L}_{2}$ stability as defined in Nešić and Dower ${ }^{25}$ (Property I3). We provide examples to demonstrate the generality and usefulness of our results.

The paper is organized as follows. Section 2 introduces the plant and assumptions placed upon it. Section 3 demonstrates boundedness of solutions of the original system (Lemma 1) and SPA stability for the fast states of the system (Corollary 1). Section 4 contains the main result of the paper. First, boundedness of solutions is proven for the observer dynamics (Corollary 2). Then, a semi-global practical ISS and $\mathcal{L}_{2} \cap \mathcal{L}_{\infty}$ stability properties are proven for the error dynamics in our main contribution (Theorem 1). Then, in Section 5 we illustrate and demonstrate the applicability of our results by presenting three classes of plants and observers for which our results hold. Although not presented here, the results cover the situation when the reduced (slow) system is such that reduced-order ${ }^{2}$, full-order ${ }^{1 / 77}$, and higher-order ${ }^{\sqrt[8]{8}}$ observers can be used to estimate the slow variables. Finally, to demonstrate the theoretical findings, we test the results on a simulation of a suspension system.

Notation: Let $\mathbb{R}=(-\infty, \infty)$ and $\mathbb{R}_{\geq 0}=[0, \infty)$. The (Euclidean) norm of a vector $x \in \mathbb{R}^{n}$ is denoted as $|x|$. We say that $s \in \mathcal{L}_{\infty}$ if $\|s\|_{\infty}<\infty$, where $\|s\|_{\infty}:=\operatorname{ess}_{\sup _{t}}|s(t)|$. We use the notation ||$s\left[t_{1}, t_{2}\right]||:=\operatorname{ess}_{\sup _{t \in\left[t_{1}, t_{2}\right]}|s(t)| \text {. The maximum }}$ (minimum) eigenvalue of a square matrix $A$ is denoted by $\lambda_{\max }\{A\}\left(\lambda_{\min }\{A\}\right)$. A function $\alpha(\cdot): \mathbb{R}_{\geq 0} \rightarrow \mathbb{R}_{\geq 0}$ is said to be of class- $\mathcal{K}(\alpha(\cdot) \in \mathcal{K})$ if it is continuous, zero at zero and strictly increasing; additionally, if $\alpha(r) \rightarrow \infty$ as $r \rightarrow \infty, \alpha(\cdot)$ is said to be a class- $\mathcal{K}_{\infty}$ function $\left(\alpha(\cdot) \in \mathcal{K}_{\infty}\right)$. A function $\sigma(\cdot)$ is said to be of class- $\mathcal{L}(\sigma(\cdot) \in \mathcal{L})$, if it is continuous, non-increasing and $\sigma(r) \rightarrow 0$ as $r \rightarrow \infty$. A function $\beta(\cdot, \cdot): \mathbb{R}_{\geq 0} \times \mathbb{R}_{\geq 0} \rightarrow \mathbb{R}_{\geq 0}$ is said to be of class- $\mathcal{K} \mathcal{L}(\beta(\cdot, \cdot) \in \mathcal{K} \mathcal{L})$, if for each fixed $s \in \mathbb{R}_{\geq 0}$, $\beta(\cdot, s) \in \mathcal{K}$ and for each fixed $r \in \mathbb{R}_{\geq 0}, \beta(r, \cdot) \in \mathcal{L}$. 


\section{2 | SINGULARLY PERTURBED PLANT}

We consider a class of plants in the following form

$$
\begin{aligned}
\dot{x} & =f_{s}(t, x, z, u(t), \epsilon), \\
\epsilon \dot{z} & =f_{f}(t, x, z, u(t), \epsilon), \\
y & =h(t, x, z, u(t), w(t), \epsilon),
\end{aligned}
$$

where $x \in \mathbb{R}^{n}$ and $z \in \mathbb{R}^{m}$ are the slow and fast states of the system respectively. The measured output is $y \in \mathbb{R}^{p}$, the perturbation parameter of the system representing the time scale separation is $\epsilon>0, u \in \mathbb{R}^{r}$ denotes the input vector and $w \in \mathbb{R}^{s}$ is the measurement noise to the system which is assumed to be bounded. The vector $u(t)$ is a measured input that may represent a control input, exogenous measured disturbances, constant or time-varying parameters or tracking signals. In the sequel, for simplicity, we will suppress the argument $t$ in the notation of the vector input $u(t)$ and in the measurement noise $w(t)$.

Remark 1. The state estimation literature deals with the development of reliable methods (observers) to accurately reconstruct the state variables of a system. In this context, accuracy is understood as the quality of the estimates to converge to the true states. Observers have a wide range of applicability, which may include modelling (identification), monitoring (fault detection), or driving (control) the system ${ }^{9}$. Hence, the observer design problem can be formulated as an open-loop or a closed-loop problem. When it is addressed in an open-loop setting, the input of the plant is understood as a known external signal. When the problem is studied from its closed-loop viewpoint, the observer is used to produce state estimates for control purposes. Here, we state our results in an open-loop setting so that the measured input $u(t)$ in (1) is regarded as an external bounded signal, which may take different meanings depending on the context. The input signal may represent a control, exogenous measured disturbances, constant or time-varying parameters or tracking signals. Although we consider an open-loop setting, we state semi-global results which cover closed-loop scenarios (i.e. when $u=u(x)$ ) under extra mild assumptions. Therefore, we address the observer design problem from its more general perspective.

Remark 2. We use the term measurement noise when we refer to an external disturbance affecting the output of the system. We study the impact of the amplitude of such a disturbance on the convergence properties of a state observer. Since we only care of the measurement noise amplitude, it can be high or low frequency. Although the study of the stochastic properties of the measurement noise is an important problem in its own right, it is outside of the scope of this manuscript. Note that the study of the noise as an stochastic quantity may be of interest in some areas of engineering. However, it is a different problem that has to be addressed separately as it requires of a different modelling framework.

Our aim is to analyse the robustness, with respect to singular perturbations, of nonlinear observers designed to estimate the slow variable $x$ of the singularly perturbed system (1), by assuming that $y$ and $u$ are available. We follow the standard procedure on linear/nonlinear observer design for singularly perturbed systems; 1) we approximate the full system (1) by two lower dimensional systems: the reduced and the boundary layer systems, 2) we then design an observer using the reduced (slow) system, and implement it on the original system. Then, we analyse the performance of the estimation error in the original system and prove that, under a set of appropriate general conditions, the aforementioned approach leads to ISS and $\mathcal{L}_{2}$ stability properties for the error dynamics. We state useful SPA convergence results for the estimation error.

The standard singular perturbations technique is the decomposition of original system (1) into lower dimensional systems associated with different time scales. Then, we set $\epsilon=0$ to obtain the following algebraic equation

$$
0=f_{f}(t, x, z, u, 0) \text {. }
$$

Assumption 1. The algebraic equation (2) has an isolated solution $z=H(t, x, u)$ that can be obtained analytically and is used to define the reduced (slow) system.

Assumption 1 is common in the singular perturbations framework since it is required to analyse the quasi-steady state behaviour of the singularly perturbed system. Moreover, we must know $H(t, x, u)$ to define the slow system which is needed to designing an observer for the slow states of the plant. Since we assume that we know $H(t, x, u)$, we substitute the isolated solution $z=H(t, x, u)$ in $(1 \mathrm{a})$ and $(1 \mathrm{c})$ at $\epsilon=0$ to obtain the reduced (slow) dynamical system

$$
\begin{aligned}
\dot{x} & =f_{s}(t, x, H(t, x, u), u, 0), \\
y_{s} & =h(t, x, H(t, x, u), u, w, 0) .
\end{aligned}
$$


Assumption 2. For the slow system $\sqrt{3}$, there exists a continuously differentiable function $V_{1}(t, x)$, class- $\mathcal{K}_{\infty}$ functions $\underline{\alpha}_{V_{1}}(\cdot)$, $\bar{\alpha}_{V_{1}}(\cdot), \alpha_{V_{1}}(\cdot), \gamma_{V_{1}}(\cdot)$, and $\zeta_{1}>0, \delta_{V_{1}} \geq 0$, such that for all $x \in \mathbb{R}^{n}, u \in \mathbb{R}^{r}, t \geq 0$

$$
\begin{aligned}
\underline{\alpha}_{V_{1}}(|x|) \leq V_{1}(t, x) & \leq \bar{\alpha}_{V_{1}}(|x|), \\
\frac{\partial V_{1}}{\partial t}+\frac{\partial V_{1}}{\partial x} f_{s}(t, x, H(t, x, u), u, 0) & \leq-\zeta_{1} \alpha_{V_{1}}^{2}(|x|)+\gamma_{V_{1}}(|u|)+\delta_{V_{1}} .
\end{aligned}
$$

Remark 3. Assumption 2 implies that, for any essentially bounded input, the system $(3)$ is globally input-to-state practically stable $26 \mid 28$. This assumption is standard in nonlinear systems since the observer design for nonlinear unbounded systems is notoriously difficult. Assumption 2 covers systems with globally stable limit cycles, i.e. systems with slow states that do not necessarily converge to the origin. For instance, the Van der Pol Oscillator ${ }^{29}$, Hamiltonian systems ${ }^{30}$, the elastic pendulum ${ }^{31}$, some biological systems $\frac{32}{}$, and so on.

To analyse the fast dynamics behaviour, we consider the change of variables $\xi=z-H(t, x, u)$. The system (1) in the new coordinates $(x, \xi)$ is represented by

$$
\begin{aligned}
\dot{x} & =f_{s}(t, x, \xi+H(t, x, u), u, \epsilon), \\
\epsilon \dot{\xi} & =f_{f}(t, x, \xi+H(t, x, u), u, \epsilon)-\epsilon\left(\frac{\partial H}{\partial t}+\frac{\partial H}{\partial x} f_{s}(t, x, \xi+H(t, x, u), u, \epsilon)+\frac{\partial H}{\partial u} \dot{u}\right), \\
y & =h(t, x, \xi+H(t, x, u), u, w, \epsilon),
\end{aligned}
$$

in which the quasi-steady-state of the fast dynamics is $\xi=0$. Consider the fast time scale $\tau$ defined as $\tau:=\frac{t-t_{0}}{\epsilon}$. Hence, in the $\tau$-time scale, the singularly perturbed system (6a)- (6b) takes the form

$$
\begin{aligned}
& \frac{d x}{d \tau}=\epsilon f_{s}(t, x, \xi+H(t, x, u), u, \epsilon), \\
& \frac{d \xi}{d \tau}=f_{f}(t, x, \xi+H(t, x, u), u, \epsilon)-\epsilon\left(\frac{\partial H}{\partial t}+\frac{\partial H}{\partial x} f_{s}(t, x, \xi+H(t, x, u), u, \epsilon)+\frac{\partial H}{\partial u} \dot{u}\right) .
\end{aligned}
$$

Setting $\epsilon=0$ freezes the variables $t=t_{0}$ and $x=x\left(t_{0}\right)$, and reduces $(7 \mathrm{~b}$ ) to the autonomous system

$$
\frac{d \xi}{d \tau}=f_{f}\left(t_{0}, x\left(t_{0}\right), \xi+H\left(t_{0}, x\left(t_{0}\right), u\right), u, 0\right) .
$$

Observe that the solutions of $(8)$ will converge to a $O(\epsilon)$ neighbourhood of the origin during the boundary layer interval. After that interval, the slowly varying parameters $(t, x)$ are not longer close enough to their initial values $\left(t_{0}, x\left(t_{0}\right)\right)$. Then, a stability property must be assumed for (8) such that its solutions remain in a neighbourhood of zero. To do so, the frozen variables $t=t_{0}$ and $x=x\left(t_{0}\right)$ must be allowed to take values in the region of the the slowly varying parameters $(t, x)$. Therefore, we rewrite (8) as follows

$$
\frac{d \xi}{d \tau}=f_{f}(t, x(t), \xi+H(t, x(t), u), u, 0),
$$

where $(t, x)$ are thought as fixed parameters. We refer to $(9)$ as the boundary layer system. For further details on how the boundary layer system is obtained, the reader can refer to Chapter 11 of Khalil ${ }^{29}$ and/or Chapter 7 of Kokotović et al ${ }^{33}$.

Assumption 3. For the Boundary Layer System $\left(9 p\right.$ there exists a Lyapunov function $W(t, x, \xi)$ and class- $\mathcal{K}_{\infty}$ functions $\underline{\alpha}_{W}(\cdot)$, $\bar{\alpha}_{W}(\cdot)$ and $\alpha_{W}(\cdot)$, and $\zeta_{3}>0$ such that for all $t, x, \xi$ we have

$$
\begin{gathered}
\underline{\alpha}_{W}(|\xi|) \leq W(t, x, \xi) \leq \bar{\alpha}_{W}(|\xi|), \\
\frac{\partial W}{\partial \xi} f_{f}(t, x, \xi+H(t, x, u), u, 0) \leq-\zeta_{3} \alpha_{W}^{2}(|\xi|) .
\end{gathered}
$$

Note that Assumption 3 implies that the boundary layer dynamics are globally asymptotically stable uniformly in $t, x$ and $u$.

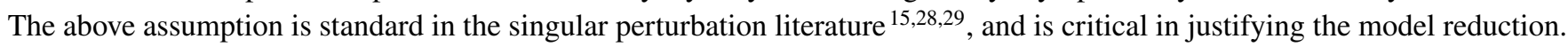

\section{3 | BOUNDEDNESS OF SOLUTIONS OF THE PLANT}

In this section, we provide a result that shows the boundedness of solutions of the system (6) under certain conditions, with a view to later using it for robustness analysis of the proposed approach to observer design. Although boundedness of solutions of the 
full system can be assumed, we state it for two reasons, 1) this result is of interest in its own right and 2) some of the assumptions we state for this result are also needed to prove much stronger conclusion on the stability of the error dynamics. In our analysis, we compute the derivatives of $V_{1}(t, x)$ and $W(t, x, \xi)$, given in Assumptions 2 and 3, along the trajectories of (6). This leads to some terms representing the interconnections between the slow and the fast dynamics. In general, those interconnection terms are sign indefinite; therefore, we need appropriate conditions to bound them to conclude boundedness of solutions.

Assumption 4. Consider $\alpha_{V_{1}}(\cdot)$ and $\alpha_{W}(\cdot)$ given in Assumptions 2 and 3 respectively. Suppose there exist non-negative constants $a_{i}(i=1,2,3)$ and $b_{i}(i=1,2,3)$, and class- $\mathcal{K}_{\infty}$ functions $\gamma_{i}(\cdot)(i=1, \ldots, 4)$, so that the following conditions hold

$$
\begin{gathered}
\left|\frac{\partial V_{1}}{\partial x}\left[f_{s}(t, x, \xi+H(t, x, u), u, \epsilon)-f_{s}(t, x, H(t, x, u), u, 0)\right]\right| \leq \epsilon a_{1} \alpha_{V_{1}}^{2}(|x|)+\epsilon \gamma_{1}(|u|) \alpha_{V_{1}}(|x|)+b_{1} \alpha_{V_{1}}(|x|) \alpha_{W}(|\xi|), \\
\left|\frac{\partial W}{\partial \xi}\left[f_{f}(t, x, \xi+H(t, x, u), u, \epsilon)-f_{f}(t, x, \xi+H(t, x, u), u, 0)\right]\right| \leq \epsilon a_{2} \alpha_{W}^{2}(|\xi|)+\epsilon \gamma_{2}(|u|) \alpha_{W}(|\xi|)+\epsilon b_{2} \alpha_{V_{1}}(|x|) \alpha_{W}(|\xi|) \\
\left|\frac{\partial W}{\partial t}-\frac{\partial W}{\partial \xi} \frac{\partial H}{\partial t}-\frac{\partial W}{\partial \xi} \frac{\partial H}{\partial u} \dot{u}+\left[\frac{\partial W}{\partial x}-\frac{\partial W}{\partial \xi} \frac{\partial H}{\partial x}\right] f_{s}(t, x, \xi+H(t, x, u), u, \epsilon)\right| \leq a_{3} \alpha_{W}^{2}(|\xi|) \\
+b_{3} \alpha_{V_{1}}(|x|) \alpha_{W}(|\xi|)+\gamma_{3}(|u|) \alpha_{W}(|\xi|)+\gamma_{4}(|\dot{u}|) \alpha_{W}(|\xi|)
\end{gathered}
$$

for all $(x, \xi) \in \mathbb{R}^{n} \times \mathbb{R}^{m}, u \in \mathbb{R}^{r}, \dot{u} \in \mathbb{R}^{r}$ and $t \geq 0$.

Remark 4. Assumption 4 can be relaxed to hold regionally or locally. Moreover, conditions in Assumption 4 can be relaxed to hold semi-globally with respect to the perturbation parameter $\epsilon$. This means that for any positive constants $\delta_{1}>0, \delta_{2}>0$, $\delta_{3}>0$ there exists $\epsilon_{a_{5}}^{*}>0$ such that $[12)$ - $(14)$ holds for all $\epsilon \in\left(0, \epsilon_{a_{5}}^{*}\right)$ and for all $|(x, \xi)| \leq \delta_{1},|u| \leq \delta_{2},|\dot{u}| \leq \delta_{2}$ and $t \geq 0$. Our proofs are such that our results can be easily extended to cover these cases.

The inequalities (12)- 14 are general and similar to the ones in Kokotovic et al $\frac{15}{4}$. These interconnection conditions are satisfied in a number of real world examples we considered; for instance, a suspension system ${ }^{29}$, a biological reactor ${ }^{10}$, a threestate SCR catalyst $\frac{13}{13}$, and so on. Note that we have also checked classes of plants for which these interconnection conditions hold. For example, the class of systems covered by the circle criterion observer ${ }^{2}$ and the class of plants in the observability canonical form $^{8}$. Moreover, the above inequalities can be verified in several examples by using quadratic-type Lyapunov functions ${ }^{34}$. A mechanical example that satisfies Assumption 4 is presented in Section 5

We now present our first result (Lemma 1) which states that, under Assumptions 1 - 4 for sufficiently small values of $\epsilon$, the singularly perturbed system (6) has bounded solutions for essentially bounded inputs with essentially bounded derivatives. Moreover, we also present a result in terms of $\mathcal{L}_{2}$ stability which guarantees that bounded energy inputs imply practical bounded solutions. Results in Lemma 1 are used later to prove some corollaries and the main result.

Lemma 1. Consider the singularly perturbed system (6). If Assumptions 1-4hold, there exists a composite Lyapunov function $V(t, x, \xi)$, class- $\mathcal{K}_{\infty}$ functions $\underline{\alpha}_{V}(\cdot), \bar{\alpha}_{V}(\cdot), \gamma_{V}(\cdot), \tilde{\gamma}_{V}(\cdot), \hat{\gamma}_{V}(\cdot)$, and $\mu_{V}>0$, such that there exists $\tilde{\epsilon}^{*}>0$ and $\alpha_{V}(\cdot) \in \mathcal{K}_{\infty}$, such that

$$
\begin{gathered}
\underline{\alpha}_{V}(|(x, \xi)|) \leq V(t, x, \xi) \leq \bar{\alpha}_{V}(|(x, \xi)|), \\
\frac{\partial V}{\partial t}+\frac{\partial V}{\partial x} f_{s}+\frac{\partial V}{\partial \xi} f_{f} \leq-\alpha_{V}(|(x, \xi)|)+\gamma_{V}(|u|)+\epsilon \tilde{\gamma}_{V}(|u|)+\epsilon \hat{\gamma}_{V}(|\dot{u}|)+\mu_{V},
\end{gathered}
$$

hold for all $\epsilon \in\left(0, \tilde{\epsilon}^{*}\right)$ and for all $(x, \xi) \in \mathbb{R}^{n} \times \mathbb{R}^{m}, u \in \mathbb{R}^{r}, \dot{u} \in \mathbb{R}^{r}$ and $t \geq 0$. Consequently, there exists $\beta_{L_{1}}(\cdot, \cdot) \in \mathcal{K} \mathcal{L}$, $\gamma_{L_{1}}(\cdot) \in \mathcal{K}_{\infty}$, class- $\mathcal{K}_{\infty}$ functions $\tilde{\gamma}_{\epsilon}(\cdot), \hat{\gamma}_{\epsilon}(\cdot)$ parametrized by $\epsilon$ (their argument is of order $O(\epsilon)$ ), and $\mu_{L_{1}}>0$, such that, for any $\hat{\Delta}_{u_{1}}>0$ and $\hat{\Delta}_{u_{2}}>0$ a

$$
|(x(t), \xi(t))| \leq \beta_{L_{1}}\left(\left|\left(x_{0}, \xi_{0}\right)\right|, t-t_{0}\right)+\gamma_{L_{1}}\left(\| u\left[t_{0}, t\right]||\right)+\tilde{\gamma}_{\epsilon}\left(\| u\left[t_{0}, t\right]||\right)+\hat{\gamma}_{\epsilon}\left(\left\|\dot{u}\left[t_{0}, t\right]\right\|\right)+\mu_{L_{1}},
$$

for all $\epsilon \in\left(0, \tilde{\epsilon}^{*}\right)$ and for all $\left(x_{0}, \xi_{0}\right) \in \mathbb{R}^{n} \times \mathbb{R}^{m},\|u\|_{\infty} \leq \hat{\Delta}_{u_{1}},\|\dot{u}\|_{\infty} \leq \hat{\Delta}_{u_{1}}$ and $t \geq t_{0} \geq 0$. Furthermore, the system (6) satisfies

$$
\int_{t_{0}}^{t} \alpha_{V}(|(x(s), \xi(s))|) d s \leq \bar{\alpha}_{V}\left(\left|\left(x_{0}, \xi_{0}\right)\right|\right)+\int_{t_{0}}^{t} \gamma_{V}(|u(s)|) d s+\epsilon \int_{t_{0}}^{t} \tilde{\gamma}_{V}(|u(s)|) d s+\epsilon \int_{t_{0}}^{t} \hat{\gamma}_{V}(|\dot{u}(s)|) d s+\mu_{V}\left(t-t_{0}\right),
$$

\footnotetext{
an the sequel, $x_{0}:=x\left(t_{0}\right)$. The same applies for the other states.
} 
for all $\epsilon \in\left(0, \tilde{\epsilon}^{*}\right),\left(x_{0}, \xi_{0}\right) \in \mathbb{R}^{n} \times \mathbb{R}^{m}$, for any input satisfying $\|u\|_{\infty} \leq \hat{\Delta}_{u_{1}},\|\dot{u}\|_{\infty} \leq \hat{\Delta}_{u_{2}},\|u\|_{\mathcal{L}_{2}} \leq \hat{\Delta}_{u_{1}},\|\dot{u}\|_{\mathcal{L}_{2}} \leq \hat{\Delta}_{u_{2}}$, and for all $t \geq t_{0} \geq 0$.

The proof for Lemma 1 is omitted since the major contribution of this work lies in the robustness analysis of the observer. Lemma 1 implies that both slow and fast states are bounded. Note that we stated ISS and $\mathcal{L}_{2} \cap \mathcal{L}_{\infty}$ stability properties with gains from the input and its derivative as $u(t)$ is regarded as a general exogenous signal to the system which can take different meanings depending on the context. Now, we state that the fast states are ultimately bounded by a constant term that one can make arbitrarily small by reducing $\epsilon$. We also show that the $\mathcal{L}_{2}$ upper bound is parametrized by $\epsilon$, which is a desired property in this framework.

Corollary 1. Consider the singularly perturbed system (6). If Assumptions 1 - 4 hold, there exists $\beta_{\xi}(\cdot, \cdot) \in \mathcal{K} \mathcal{L}$, such that for any $\tilde{\Delta}>0, \tilde{\Delta}_{u_{1}}>0, \tilde{\Delta}_{u_{2}}>0$ and $\tilde{\mu}>0$, there exists $\bar{\epsilon}^{*}>0$, such that

$$
|\xi(t)| \leq \max \left\{\beta_{\xi}\left(\left|\xi_{0}\right|, \frac{t-t_{0}}{\epsilon}\right), \tilde{\mu}\right\},
$$

for all $\epsilon \in\left(0, \bar{\epsilon}^{*}\right),\left|\left(x_{0}, \xi_{0}\right)\right| \leq \tilde{\Delta},\|u\|_{\infty} \leq \tilde{\Delta}_{u_{1}},\|\dot{u}\|_{\infty} \leq \tilde{\Delta}_{u_{2}}$ and $t \geq t_{0} \geq 0$. Consequently, there exists a time $\bar{T}^{*}>0$ such that

$$
|\xi(t)| \leq \tilde{\mu},
$$

for all $\epsilon \in\left(0, \bar{\epsilon}^{*}\right),\left|\left(x_{0}, \xi_{0}\right)\right| \leq \tilde{\Delta},\|u\|_{\infty} \leq \tilde{\Delta}_{u_{1}},\|\dot{u}\|_{\infty} \leq \tilde{\Delta}_{u_{2}}$ and $t \geq \epsilon \bar{T}^{*}+t_{0}>0$. Furthermore, there exists $\alpha_{W_{c}}(\cdot) \in \mathcal{K}_{\infty}$, such that for the given $\tilde{\Delta}>0, \tilde{\Delta}_{u_{1}}>0, \tilde{\Delta}_{u_{2}}>0$ and $\tilde{\mu}>0$ there exist $\bar{\epsilon}_{\mathcal{L}_{2}}^{*}>0$, such that

$$
\int_{t_{0}}^{t} \alpha_{W}(|\xi(s)|) d s \leq \epsilon \alpha_{W_{c}}\left(\left|\xi_{0}\right|\right)+\tilde{\mu}\left(t-t_{0}\right),
$$

for all $\epsilon \in\left(0, \bar{\epsilon}_{\mathcal{L}_{2}}^{*}\right),\left|\left(x_{0}, \xi_{0}\right)\right| \leq \tilde{\Delta}$, for any input satisfying $\|u\|_{\infty} \leq \tilde{\Delta}_{u_{1}},\|\dot{u}\|_{\infty} \leq \tilde{\Delta}_{u_{2}},\|u\|_{\mathcal{L}_{2}} \leq \tilde{\Delta}_{u_{1}},\|\dot{u}\|_{\mathcal{L}_{2}} \leq \tilde{\Delta}_{u_{2}}$, and for all $t \geq t_{0} \geq 0$.

The proof for Corollary 1 is omitted too. The proof relies on the analysis of the fast dynamics by considering the Lyapunov function for the boundary-layer system and Lemma 1. Note that the statement of the corollary implies that the ultimate bound in (19) and (20) can be made arbitrarily small. To do so, it is required to reduce $\epsilon$, i.e., the magnitude of the ultimate bound determines the maximum value that the perturbation parameter can take. The properties given by Corollary 1 are exploited later in the proof of our main result.

\section{4 | MAIN RESULT}

In this section, we analyse the performance of a nonlinear observer designed for the reduced system (3) and implemented on the system (6). We do not estimate the fast states of the system. We study the robustness of the observer with respect to singular perturbations due to the neglected fast dynamics. We provide a general set of conditions to cover a large class of plant models and observers of general dimension. Since we assume that a nonlinear observer exists, our results are prescriptive. Our conditions justify the use of a broader class of observers than results in Kazantzis et al ${ }^{1]}$. While Kazantzis et al ${ }^{1]}$ considers nonlinear plants where the slow part of the model satisfies a Lipschitz condition and the fast dynamics and the output of the system are linear, here we deal with a more general class of nonlinear plants. Moreover, whilst Kazantzis et al ${ }^{11}$ only works with a nonlinear Luenbergertype observer that exhibits a linear error dynamics for the reduced system, our results can cover a number of nonlinear observers including reduced-order ${ }^{[2}$, full-order ${ }^{[1 / 7}$, and higher-order ${ }^{[8]}$ observers.

We now assume that a nonlinear observer is designed for the reduced system (3). So, consider the following dynamical system called the observer

$$
\begin{aligned}
& \dot{\chi}=f_{o}\left(t, \chi, y_{s}, u\right), \\
& \hat{x}=h_{o}(t, \chi, u),
\end{aligned}
$$

where $\chi \in \mathbb{R}^{q}$ is the observer's state, $\hat{x} \in \mathbb{R}^{n}$ is the observer's output and an estimate of $x$ (slow variable), $y_{s}$ and $u$ are the output and input of the nonlinear reduced system (3). Note that, in general, $q$ is arbitrary and not necessarily equal to $n$. The 
class of observers that we consider in (22) covers observers of general dimension. Existing results on nonlinear observer design for singularly perturbed systems only cover a Luenberger-type full-order observer $\stackrel{1}{1}$.

Remark 5. In the case of reduced-order observers, the observer dynamics are generally designed on the basis of an auxiliary subsystem. Then, for reduced-order observers, the output of the observer (22b) may depend on the output of the system $\left(y_{s}\right)$. We allow this dependency only if $y_{s}$ does not appear in the estimation error. We demonstrate in Section 5 that the reduced-order circle criterion observer ${ }^{2}$ satisfies this condition.

Assumption 5. The map $h_{o}(t, \chi, u):[0, \infty) \times \mathbb{R}^{q} \times \mathbb{R}^{r} \rightarrow \mathbb{R}^{n}$ is a continuously differentiable function in all its arguments.

Remark 6. Assumption 5 implies that $h_{o}(t, \chi, u), \partial h_{o} / \partial \chi$ and $\partial h_{o} / \partial u$ are continuous. Consider the variable $\hat{\chi}=[\chi, u]^{T}$, it follows from Lemma 3.2 in Khali ${ }^{29}$ that for any $\Delta_{1}>0$ and $\Delta_{2}>0$ there exists $L_{0}>0$ so that when $\left|\partial h_{o} / \partial \hat{\chi}\right| \leq L_{o}$ for all $\chi$ and $u$ such that $|\chi| \leq \Delta_{1}$ and $|u| \leq \Delta_{2}$, there exists $L>0$ such that $\left|\partial h_{o} / \partial \chi\right| \leq L$. We have verified that Assumption 5 holds for reduced-order ${ }^{2}$, full-order ${ }^{[1+7]}$, and higher-order ${ }^{[8}$ observers.

Define the estimation error as $e=\hat{x}-x$. Note that the error dynamics for the observer designed for the reduced system (3) are given by

$$
\dot{e}=f_{e}\left(t, x, \chi, e, H(t, x, u), y_{s}, u, \dot{u}, 0\right) \text {. }
$$

where

$$
f_{e}\left(t, x, \chi, e, H(t, x, u), y_{s}, u, \dot{u}, 0\right)=\frac{\partial h_{o}}{\partial t}+\frac{\partial h_{o}}{\partial \chi} f_{o}\left(t, \chi, y_{s}, u\right)+\frac{\partial h_{o}}{\partial u} \dot{u}-f_{s}(t, x, H(t, x, u), u, 0) .
$$

Remark 7. For reduced-order observers relying on Remark 5, the error dynamics are defined by considering an auxiliary system used to construct the observer dynamics and the observer model itself. Later in Section 5 , we illustrate this statement and Remark 5 via a reduced-order circle criterion observer ${ }^{2}$.

Assumption 6. For the error dynamics in (23), there exists a continuously differentiable function $V_{3}(t, e)$, class- $\mathcal{K}_{\infty}$ functions $\underline{\alpha}_{V_{3}}(\cdot), \bar{\alpha}_{V_{3}}(\cdot), \alpha_{V_{3}}(\cdot), \gamma_{V_{3}}(\cdot)$, and $\zeta_{2}>0, \hat{\zeta}_{2}>0$, such that for all $(x, e) \in \mathbb{R}^{n} \times \mathbb{R}^{n}, u \in \mathbb{R}^{r}, t \geq 0$

$$
\begin{aligned}
& \underline{\alpha}_{V_{3}}(|e|) \leq V_{3}(t, e, x, \chi) \leq \bar{\alpha}_{V_{3}}(|e|), \\
& \frac{\partial V_{3}}{\partial t}+\frac{\partial V_{3}}{\partial e} f_{e}\left(t, x, \chi, e, H(t, x, u), y_{s}, u, \dot{u}, 0\right)+\frac{\partial V_{3}}{\partial x} f_{s}(t, x, H(t, x, u), u, 0)+\frac{\partial V_{3}}{\partial \chi} f_{o}\left(t, \chi, y_{s}, u\right) \leq \\
&\left|\frac{\partial V_{3}}{\partial e}\right| \leq \hat{\zeta}_{2} \alpha_{V_{3}}(|e|) .-\zeta_{2} \alpha_{V_{3}}^{2}(|e|)+\gamma_{V_{3}}(|w|),
\end{aligned}
$$

The reduced-order observer presented in $\operatorname{Arcak}^{[2}$, the full-order observers introduced in ${ }^{[1,7]}$, and the higher-order observer in Astolfi and Marconi ${ }^{8}$ satisfy Assumption 6 . It is observed that in the case of reduced-order observers $e \in \mathbb{R}^{q}$. Note that condition (26) is common when one wants to use a Lyapunov function to prove robustness of a stability property, which is the case in this work. Since we only deal with the amplitude of the measurement noise, we analyse its effect on the observer performance by introducing a nonlinear gain from the measurement noise to the state estimation error in 25 .

Remark 8. It can be proven that Assumption 6 implies a boundedness of solutions property for the observer dynamics when $q=n$, i.e., the observer is of full-order. This can also be shown for reduced-order observers like the one in Section 5.2 of Arcak $^{2}$. Note that the estimation error completely captures the behaviour of the observer state in full-order observers and some particular reduced-order observers. To show a boundedness property from Assumption 6 one can use $V_{3}(t, \chi)$ as a candidate Lyapunov function for the observer dynamics; then, by using some mild conditions on $f_{s}$ and using $(26)$ the result can be proven.

When $q \neq n$ (higher order and reduced order observers), it might be complicated or even impossible to show boundedness of solutions of the observer dynamics by just using Assumption 6 Hence, we need to assume that the observer has bounded solutions since we need such a property to prove a robustness result for observers of general dimension.

Assumption 7. For the observer dynamics 22 , there exist class- $\mathcal{K}_{\infty}$ functions $\alpha_{o_{1}}(\cdot), \alpha_{o_{2}}(\cdot)$, and $\alpha_{o_{3}}(\cdot)$, such that for all $\chi_{0} \in \mathbb{R}^{q}$, $y, u \in \mathcal{L}_{\infty}, t \geq t_{0} \geq 0$

$$
|\chi(t)| \leq \alpha_{o_{1}}\left(\left|\chi_{0}\right|\right)+\alpha_{o_{2}}\left(|| y||_{\infty}\right)+\alpha_{o_{3}}\left(\left.|| u\right|_{\infty}\right) .
$$


Moreover, if $u(t)$ and $y(t)$ are essentially bounded input signals to the observer dynamics [22], there exist $\alpha_{o_{4}}(\cdot), \alpha_{o_{5}}(\cdot), \alpha_{o_{6}}(\cdot), \alpha_{o_{7}}(\cdot) \in \mathcal{K}_{\infty}$, such that

$$
\int_{t_{0}}^{t} \alpha_{o_{4}}(|\chi(\tau)|) d \tau \leq \alpha_{o_{5}}\left(\left|\chi_{0}\right|\right)+\int_{t_{0}}^{t} \alpha_{o_{6}}(|y(\tau)|) d \tau+\int_{t_{0}}^{t} \alpha_{o_{7}}(|u(\tau)|) d \tau .
$$

To prove the robustness of the observer (22), its dynamics must have some kind of boundedness of solutions property when implemented on the original system. We use Assumption 7 to show that the observer states are ultimately bounded when we apply it to the original singularly perturbed system. Moreover, we prove that the observer states has a practical $\mathcal{L}_{2}$ stability property in the sense of Property I3 in Nešić and Dower ${ }^{25}$.

As mentioned above, the observer designed for the slow system (3) must be implemented on the original system (1). Due to the influence of the perturbation parameter $\epsilon$ and the fast state $\xi$, the observer and error dynamics are given by

$$
\begin{aligned}
\dot{\chi} & =f_{o}(t, \chi, y, u), \\
\dot{e} & =f_{e}(t, x, \chi, e, \xi+H(t, x, u), y, u, \dot{u}, \epsilon),
\end{aligned}
$$

where占

$$
f_{e}=\frac{\partial h_{o}}{\partial t}+\frac{\partial h_{o}}{\partial \chi} f_{o}(t, \chi, y, u)+\frac{\partial h_{o}}{\partial u} \dot{u}-f_{s}(t, x, \xi+H(t, x, u), u, \epsilon) .
$$

Note that the extended state $(x, \chi, e, \xi)$ represents the interconnection between the system (6), and the observer and error dynamics in 29]. Hence, we need to analyse the estimation error performance in the full extended interconnected system given by

$$
\begin{aligned}
\dot{x} & =f_{s}(t, x, \xi+H(t, x, u), u, \epsilon), \\
\dot{\chi} & =f_{o}(t, \chi, y, u) \\
\dot{e} & =f_{e}(t, x, \chi, e, \xi+H(t, x, u), y, u, \dot{u}, \epsilon) \\
\epsilon \dot{\xi} & =f_{f}(t, x, \xi+H(t, x, u), u, \epsilon)-\epsilon \frac{\partial H}{\partial t}-\epsilon \frac{\partial H}{\partial x} f_{s}(t, x, \xi+H(t, x, u), u, \epsilon)-\epsilon \frac{\partial H}{\partial u} \dot{u}, \\
y & =h(t, x, \xi+H(t, x, u), u, w, \epsilon) .
\end{aligned}
$$

Note that the observer dynamics are in a cascade with the original state $(x, \xi)$, while the error dynamics are in cascade with the extended state $(x, \chi, \xi)$. We exploit these properties to conclude our main result.

Assumption 8. Consider the output of the system $(30)$. There exists class- $\mathcal{K}_{\infty}$ functions $\alpha_{y}(\cdot), \gamma_{y}(\cdot)$ and $\gamma_{w}(\cdot)$, such that, for any $\hat{\Delta}>0, \hat{\Delta}_{u_{1}}>0$ and $\hat{\Delta}_{w}>0$, there exists $\epsilon_{y}$ such that

$$
|h(t, x, \xi+H(t, x, u), u, w, \epsilon)| \leq \alpha_{y}(|(x, \xi)|)+\gamma_{y}(|u|)+\gamma_{w}(|w|) .
$$

for all $\epsilon \in\left(0, \epsilon_{y}\right)$ and for all $|(x, \xi)| \leq \hat{\Delta},|u| \leq \hat{\Delta}_{u_{1}},|w| \leq \hat{\Delta}_{w}$ and $t \geq 0$.

Assumption 8 is a mild assumption that allows us to show in Corollary 2 that the solutions of the observer are bounded when the observer is implemented on the original system. Observe that any continuous map $h$ that is zero at zero satisfies our assumption, see Sontag 26 . We use Assumption 8 in the proof of the main result to bound terms related to the output of the observer.

Corollary 2. Consider the observer dynamics $30 \mathrm{~b}$ ). If Assumptions 1 - $-5 \mid 7$ and 8 hold, there exists a class- $\mathcal{K}_{\infty}$ function $\alpha_{c_{1}}\left({ }^{\circ}\right)$, such that for any $\bar{\Delta}>0, \bar{\Delta}_{u_{1}}>0, \bar{\Delta}_{u_{2}}>0$, and $\bar{\Delta}_{w} \geq 0$ there exists $\hat{\epsilon}^{*}>0$ and $\Upsilon>0$ such that

$$
|\chi(t)| \leq \alpha_{c_{1}}\left(\left|\chi_{0}\right|\right)+\Upsilon
$$

for all $\epsilon \in\left(0, \hat{\epsilon}^{*}\right)$ and for all $\left|\left(x_{0}, \xi_{0}, \chi_{0}\right)\right| \leq \bar{\Delta},\|u\|_{\infty} \leq \bar{\Delta}_{u_{1}},\|\dot{u}\|_{\infty} \leq \bar{\Delta}_{u_{2}},\|w\|_{\infty} \leq \bar{\Delta}_{w}$ and $t \geq t_{0} \geq 0$. Furthermore, there exist class- $\mathcal{K}_{\infty}$ functions $\alpha_{c_{2}}(\cdot), \alpha_{c_{3}}(\cdot)$, such that for the given $\bar{\Delta}>0, \bar{\Delta}_{u_{1}}>0, \bar{\Delta}_{u_{2}}>0$, and $\bar{\Delta}_{w} \geq 0$ there exists $\hat{\epsilon}^{*}>0$

bIn the sequel, when needed, we would suppress arguments of some functions to simplify the notation. 
and $\Upsilon_{\mathcal{L}_{2}}>0$ such that

$$
\int_{t_{0}}^{t} \alpha_{c_{2}}(|\chi(\tau)|) d \tau \leq \alpha_{c_{3}}\left(\left|\chi_{0}\right|\right)+\Upsilon_{\mathcal{L}_{2}}\left(t-t_{0}\right),
$$

for all $\epsilon \in\left(0, \hat{\epsilon}^{*}\right),\left|\left(x_{0}, \xi_{0}, \chi_{0}\right)\right| \leq \bar{\Delta}$, for any input satisfying $\|u\|_{\infty} \leq \bar{\Delta}_{u_{1}},\|\dot{u}\|\left\|_{\infty} \leq \bar{\Delta}_{u_{2}},\right\| u\left\|_{\mathcal{L}_{2}} \leq \bar{\Delta}_{u_{1}},\right\| \dot{u} \|_{\mathcal{L}_{2}} \leq \bar{\Delta}_{u_{2}}$, for any $\|w\|_{\infty} \leq \Delta_{w},\|w\|_{\mathcal{L}_{2}} \leq \Delta_{w}$ and for all $t \geq t_{0} \geq 0$.

The proof for Corollary 2 is omitted. Note that the above result implies that the states of the observer will remain bounded even under the influence of the fast variables and the perturbation parameter. Since we study the stability property of the error dynamics when the observer is implemented on the original system, we compute the derivative of $V_{3}(t, e, x, \chi)$ along the solutions of $(30)$ in our main proof. This leads to interconnection terms which, in general, are of sign indefinite. So, we need conditions to bound those terms.

Assumption 9. Consider $\alpha_{V_{1}}(\cdot), \alpha_{W}(\cdot)$ and $\alpha_{V_{3}}(\cdot)$ given in Assumptions 2, 3 and 6 respectively. Suppose there exist non-negative constants $a_{i}$ and $b_{i}(i=4, \ldots, 7)$, and class- $\mathcal{K}_{\infty}$ functions $\gamma_{5}(\cdot)$ and $\gamma_{6}(\cdot)$, so that the following conditions hold

$$
\begin{aligned}
\left|\frac{\partial V_{3}}{\partial x}\left[f_{s}(t, x, \xi+H(t, x, u), u, \epsilon)-f_{s}(t, x, H(t, x, u), u, 0)\right]\right| & \leq \epsilon a_{4} \alpha_{V_{1}}(|x|) \alpha_{V_{3}}(|e|)+\epsilon \gamma_{5}(|u|) \alpha_{V_{3}}(|e|) \\
& +b_{4} \alpha_{V_{3}}(|e|) \alpha_{W}(|\xi|), \\
\left|\frac{\partial V_{3}}{\partial \chi}\left[f_{o}(t, \chi, y, u)-f_{o}\left(t, \chi, y_{s}, u\right)\right]\right| & \leq \epsilon a_{5} \alpha_{V_{1}}(|x|) \alpha_{V_{3}}(|e|)+b_{5} \alpha_{V_{3}}(|e|) \alpha_{W}(|\xi|), \\
\left|\frac{\partial V_{3}}{\partial e}\left[f_{s}(t, x, \xi+H(t, x, u), u, \epsilon)-f_{s}(t, x, H(t, x, u), u, 0)\right]\right| & \leq \epsilon a_{6} \alpha_{V_{1}}(|x|) \alpha_{V_{3}}(|e|)+\epsilon \gamma_{6}(|u|) \alpha_{V_{3}}(|e|) \\
& +b_{6} \alpha_{V_{3}}(|e|) \alpha_{W}(|\xi|), \\
\left|\frac{\partial V_{3}}{\partial e}\left[f_{o}(t, \chi, y, u)-f_{o}\left(t, \chi, y_{s}, u\right)\right]\right| & \leq \epsilon a_{7} \alpha_{V_{3}}(|e|) \alpha_{V_{1}}(|x|)+b_{7} \alpha_{V_{3}}(|e|) \alpha_{W}(|\xi|) .
\end{aligned}
$$

for all $(x, \xi, e, \chi) \in \mathbb{R}^{n} \times \mathbb{R}^{m} \times \mathbb{R}^{n} \times \mathbb{R}^{q}, u \in \mathbb{R}^{r}, \dot{u} \in \mathbb{R}^{r}$ and $t \geq 0$.

Remark 9. Assumption 9 can be relaxed to hold regionally or locally. Moreover, conditions in Assumption 9 can be relaxed to hold semi-globally with respect to the perturbation parameter $\epsilon$. This means that for any positive constants $\hat{\delta}_{1}>0, \hat{\delta}_{2}>0$, $\hat{\delta}_{3}>0$ there exists $\epsilon_{a_{10}}^{*}>0$ such that 34$]-37$ holds for all $\epsilon \in\left(0, \epsilon_{a_{10}}^{*}\right)$ and for all $|(x, \xi)| \leq \hat{\delta}_{1},|u| \leq \hat{\delta}_{2},|\dot{u}| \leq \hat{\delta}_{2}$ and $t \geq 0$. Our proofs are such that our results can be easily extended to cover these cases.

Remark 10. For reduced-order observers that satisfy conditions in Remarks 5 and 7, the notation on all of the above assumptions and definitions must be slightly modified. However, these modifications do not affect the essence of the assumptions and the results.

Our goal is to ensure that the observer designed for the reduced model (3) works properly when applied on the full system (1). Corollary 2 states that, under a general set of assumptions, the observer is semi-globally ultimately bounded. In the next subsection, we use that result and the results in the previous section to prove that the estimation error is semi-global practical ISS stable. We also prove a $\mathcal{L}_{2} \cap \mathcal{L}_{\infty}$ stability property for the error dynamics. Moreover, we show through a useful result that, in the absence of measurement noise, the error dynamics are SPA stable and that the ultimate bound for the error dynamics can be reduced by reducing $\epsilon$.

\section{1 | Robustness Analysis}

We now present Theorem 1 which states that the error dynamics exhibit a semi-global practical ISS and a $\mathcal{L}_{2} \cap \mathcal{L}_{\infty}$ stability properties. Our proof focuses on the convergence of the estimation error while the other states in (30) are bounded. It is crucial for our proof to take into account that the error dynamics are in cascade with the original system and the observer dynamics. The next theorem summarises our main result. 
Theorem 1. Consider the singularly perturbed system 30 . If Assumptions 1 - 9 hold, there exists $\beta_{T_{1}}(\cdot, \cdot) \in \mathcal{K} \mathcal{L}$ and $\gamma_{T_{1}}(\cdot) \in \mathcal{K}_{\infty}$, such that for any $\Delta>0, \Delta_{u_{1}}>0, \Delta_{u_{2}}>0, \Delta_{w}>0$ and $\mu>0$, there exists $\mu_{T_{1}}=\mu_{T_{1}}(\mu)>0$ and $\epsilon^{*}>0$ such that

$$
|e(t)| \leq \beta_{T_{1}}\left(\left|\left(x_{0}, \xi_{0}, e_{0}\right)\right|, t-t_{0}\right)+\gamma_{T_{1}}\left(\| w\left[t_{0}, t\right]||\right)+\mu_{T_{1}}+\mu,
$$

for all $\epsilon \in\left(0, \epsilon^{*}\right)$, and for all $\left|\left(x_{0}, \xi_{0}, \chi_{0}, e_{0}\right)\right| \leq \Delta,\|u\|_{\infty} \leq \Delta_{u_{1}},\|\dot{u}\|_{\infty} \leq \Delta_{u_{2}},\|w\|_{\infty} \leq \Delta_{w}$, and $t \geq t_{0} \geq 0$. Furthermore, there exists $\bar{\beta}_{T_{1}}(\cdot, \cdot) \in \mathcal{K} \mathcal{L}$ and $\bar{\gamma}_{T_{1}}(\cdot) \mathcal{K}_{\infty}$, such that for the given $\Delta>0, \Delta_{u_{1}}>0, \Delta_{u_{2}}>0, \Delta_{w}>0$ and $\mu>0$, there exists $T^{*}>0$ and $\epsilon^{*}>0$ such that

$$
|e(t)| \leq \bar{\beta}_{T_{1}}\left(\left|e_{0}\right|, t-t_{0}\right)+\bar{\gamma}_{T_{1}}\left(\left\|w\left[t_{0}, t\right]\right\|\right)+\mu,
$$

for all $\epsilon \in\left(0, \epsilon^{*}\right),\left|\left(x_{0}, \xi_{0}, \chi_{0}, e_{0}\right)\right| \leq \Delta,\|u\|_{\infty} \leq \Delta_{u_{1}},\|\dot{u}\|_{\infty} \leq \Delta_{u_{2}},\|w\|_{\infty} \leq \Delta_{w}$, and $t \geq \epsilon T^{*}+t_{0}$. In addition, there exists $k_{T_{1}}>0$ and $\alpha_{T_{1}}(\cdot), \bar{\alpha}_{T_{1}}(\cdot) \in \mathcal{K}_{\infty}$ such that for the given $\Delta>0, \Delta_{u_{1}}>0, \Delta_{u_{2}}>0, \Delta_{w}>0$ and $\mu>0$, there exists $\mu_{\mathcal{L}_{2}}=\mu_{\mathcal{L}_{2}}(\mu)>0$ and $\epsilon_{\mathcal{L}_{2}}^{*}>0$ such that

$$
\int_{t_{0}}^{t} \alpha_{V_{3}}^{2}(|e(\tau)|) d \tau \leq \alpha_{T_{1}}\left(\left|\left(x_{0}, \xi_{0}, e_{0}\right)\right|\right)+\epsilon \bar{\alpha}_{T_{1}}\left(\left|\left(x_{0}, \xi_{0}, e_{0}\right)\right|\right)+k_{T_{1}} \int_{t_{0}}^{t} \gamma_{V_{3}}(|w(\tau)|) d \tau+\mu_{\mathcal{L}_{2}}\left(t-t_{0}\right)+\mu\left(t-t_{0}\right),
$$

for all $\epsilon \in\left(0, \epsilon_{\mathcal{L}_{2}}^{*}\right),\left|\left(x_{0}, \xi_{0}, \chi_{0}, e_{0}\right)\right| \leq \Delta$, for any input satisfying $\|u\|_{\infty} \leq \Delta_{u_{1}},\|\dot{u}\|_{\infty} \leq \Delta_{u_{2}},\|u\|_{\mathcal{L}_{2}} \leq \Delta_{u_{1}},\|\dot{u}\|_{\mathcal{L}_{2}} \leq \Delta_{u_{2}}$, for any $\|w\|_{\infty} \leq \Delta_{w},\|w\|_{\mathcal{L}_{2}} \leq \Delta_{w}$ and for all $t \geq t_{0} \geq 0$. Furthermore, there exists $k_{T_{1}}>0$ and $\alpha_{T_{1}}(\cdot) \in \mathcal{K}_{\infty}$ such that for the given $\Delta>0, \Delta_{u_{1}}>0, \Delta_{u_{2}}>0, \Delta_{w}>0$ and $\mu>0$, there exists $T^{*}>0$ and $\epsilon_{\mathcal{L}_{2}}^{*}>0$ such that

$$
\int_{t_{0}}^{t} \alpha_{V_{3}}^{2}(|e(\tau)|) d \tau \leq \alpha_{T_{1}}\left(\left|e_{0}\right|\right)+k_{T_{1}} \int_{t_{0}}^{t} \gamma_{V_{3}}(|w(\tau)|) d \tau+\mu\left(t-t_{0}\right)
$$

for all $\epsilon \in\left(0, \epsilon_{\mathcal{L}_{2}}^{*}\right),\left|\left(x_{0}, \xi_{0}, \chi_{0}, e_{0}\right)\right| \leq \Delta$, for any input satisfying $\|u\|_{\infty} \leq \Delta_{u_{1}},\|\dot{u}\|_{\infty} \leq \Delta_{u_{2}},\|u\|_{\mathcal{L}_{2}} \leq \Delta_{u_{1}},\|\dot{u}\| \|_{\mathcal{L}_{2}} \leq \Delta_{u_{2}}$, for any $\|w\|_{\infty} \leq \Delta_{w},\|w\|_{\mathcal{L}_{2}} \leq \Delta_{w}$ and for all $t \geq \epsilon T^{*}+t_{0}$.

The proof of Theorem 1 is given in Appendix A Note that in the absence of measurement noise, stronger conclusions can be obtained as an immediate consequence of Theorem 1 . The next corollary presents these sharper results.

Corollary 3. Consider the singularly perturbed system [30. Let Assumptions 1 - 9 hold and assume that $w(t)=0$ for all $t \geq t_{0} \geq 0$. Then, there exists $\beta_{c}(\cdot, \cdot) \in \mathcal{K} \mathcal{L}$ such that for any $\Delta>0, \Delta_{u_{1}}>0, \Delta_{u_{2}}>0$ and $\mu>0$, there exists $T^{*}>0$ and $\epsilon^{*}>0$ such that

$$
|e(t)| \leq \beta_{c}\left(\left|e_{0}\right|, t-t_{0}\right)+\mu,
$$

for all $\epsilon \in\left(0, \epsilon^{*}\right),\left|\left(x_{0}, \xi_{0}, \chi_{0}, e_{0}\right)\right| \leq \Delta,\|u\|_{\infty} \leq \Delta_{u_{1}},\|\dot{u}\|_{\infty} \leq \Delta_{u_{2}}$, and $t \geq \epsilon T^{*}+t_{0}$. Furthermore, there exists $\alpha_{c}(\cdot) \in \mathcal{K}_{\infty}$ such that for the given $\Delta>0, \Delta_{u_{1}}>0, \Delta_{u_{2}}>0$ and $\mu>0$, there exists $T^{*}>0$ and $\epsilon_{\mathcal{L}_{2}}^{*}>0$ such that

$$
\int_{t_{0}}^{t} \alpha_{V_{3}}^{2}(|e(\tau)|) d \tau \leq \alpha_{c}\left(\left|e_{0}\right|\right)+\mu\left(t-t_{0}\right)
$$

for all $\epsilon \in\left(0, \epsilon_{\mathcal{L}_{2}}^{*}\right),\left|\left(x_{0}, \xi_{0}, \chi_{0}, e_{0}\right)\right| \leq \Delta$, for any input satisfying $\|u\|_{\infty} \leq \Delta_{u_{1}},\|\dot{u}\|\left\|_{\infty} \leq \Delta_{u_{2}},\right\| u\left\|_{\mathcal{L}_{2}} \leq \Delta_{u_{1}},\right\| \dot{u} \|_{\mathcal{L}_{2}} \leq \Delta_{u_{2}}$, and for all $t \geq \epsilon T^{*}+t_{0}$.

The proof is omitted since the result follows directly from Theorem 1.

Remark 11. Corollary 3 implies a SPA stability property for the error dynamics in the absence of measurement noise. It is semiglobal because the result holds for a given set of initial conditions and bounded inputs with bounded derivatives. It is practical in the perturbation parameter because one can make $\mu$ arbitrarily small by reducing $\epsilon$. And it is asymptotical because of the class$\mathcal{K} \mathcal{L}$ function $\beta_{e}(\cdot, \cdot)$. These results (Theorem 1 and Corollary 3 ) imply important robustness properties for a large class of plants and observers. So, we can choose any existing observer, satisfying this framework, to estimate the slow states of a singularly perturbed system that satisfies the given assumptions. 
Remark 12. The global and semi-global assumptions for the boundary layer and reduced systems, and for the observer and error dynamics can be relaxed. If all assumptions hold on appropriate bounded sets, the results hold in a given region defined by those sets. Moreover, our approach is such that local results can easily be stated if the assumptions are relaxed to hold locally.

Remark 13. If the fast dynamics (1b) do not depend on the input $u$, there is no need of any conditions on $\dot{u}$. Moreover, the results would not depend on $\dot{u}$ either.

Remark 14. Theorem 1 and Corollary 3 cover and generalise results in Cuevas et al ${ }^{22}$ since any system and any observer that satisfy the assumptions in there also satisfy the assumptions in this manuscript. Moreover, here we have considered a more general class of plants and observers than those in Cuevas et al ${ }^{22}$. Note that SPA results for full-order observers are presented in Cuevas et al ${ }^{[22}$ where we do not consider the presence of measurement noise.

Remark 15. Since we deliver semi-global results, we can relate the estimated states with the input to cover the observer design problem for control purposes. Suppose that in a closed-loop setting the input is a function of the state, i.e. $u=u(x)$. Then, we have that $\dot{u}=\frac{\partial u}{\partial x} \dot{x}=\frac{\partial u}{\partial x} f$. Then, semi-global bounds on $u$ and $\dot{u}$ become semi-global bounds on $u(x)$ and $\frac{\partial u}{\partial x} f$ which lead to semi-global bounds on $x$ only if $u, \frac{\partial u}{\partial x}$ and $f$ are continuous and zero at zero, which is not very restrictive.

\section{5 | APPLICATIONS}

In Cuevas et al ${ }^{23}$, we illustrated the applicability of our results presented in Cuevas et al ${ }^{22}$. We showed that those results apply for a class of systems with reduced order models for which the circle criterion observer in $\operatorname{Arcak}^{[2}$ can be designed. Moreover, we demonstrated that results in Cuevas et al ${ }^{[22}$ cover conclusions in Kazantzis et al ${ }^{1]}$.

Results in Kazantzis et al ${ }^{11}$ apply for nonlinear singularly perturbed systems with linear fast dynamics, linear output and slow dynamics satisfying a Lipschitz condition. Their approach only allows to obtain conclusions for a specific Luenbergertype nonlinear observer. We have proven in Cuevas et al ${ }^{23}$ that our results in Cuevas et a ${ }^{[22}$ cover those ones in Kazantzis et al ${ }^{1]}$. Therefore, relying on Remark 14, this manuscript covers results in Kazantzis et al ${ }^{1}{ }^{1}$ too. This work generalises findings in Kazantzis et al ${ }^{1]}$ because we deal with a general class of systems and estimators that covers several classes of plants and observers. Moreover, we have studied the robustness of the error dynamics with respect to measurement noise within the singular perturbation framework.

In the following subsections, we demonstrate that our results cover at least other three classes of plants and three nonlinear observers (one full-order ${ }^{[4}$, one reduced-order ${ }^{2]}$ and one higher-order ${ }^{[8}$ observer). Moreover, we illustrate our results through one numerical example.

\section{1 | Circle Criterion Observer}

In this section, we consider a class of singularly perturbed plants where the reduced (slow) model takes the form in which results from Chong et al ${ }^{4}$ can be applied to design a full-order observer. This class of plants is covered by the general model (1). Note that this class of systems and the observer are not covered by results in Kazantzis et al ${ }^{11}$. Consider the class of plants with the following nonlinear singularly perturbed form

$$
\begin{aligned}
\dot{x} & =A x+B z+G \gamma\left(F_{1} x+F_{2} z\right)+\sigma(y, u), \\
\epsilon \dot{z} & =M_{1} x+M_{2} z, \\
y & =C_{1} x+C_{2} z+D w,
\end{aligned}
$$

where the state vector $x \in \mathbb{R}^{n}$ corresponds to the slow state, $z \in \mathbb{R}^{m}$ is the fast state, $y \in \mathbb{R}^{p}$ is the measured output variable, $u \in \mathbb{R}^{r}$ is the control input, $w$ is the measurement noise, $\epsilon$ is the perturbation parameter of the process, $\gamma(\cdot)=\left[\gamma_{1}(\cdot), \cdots, \gamma_{n_{\gamma}}(\cdot)\right]^{T}$ is a nondecreasing locally Lipschitz function, and $A, B, F_{1}, F_{2}, G, C_{1}, C_{2}, D, M_{1}$ and $M_{2}$ are matrices of appropriate dimensions. We require a linear dynamics in (44b) for two reasons: 1) it is easier to compute the slow manifold, and 2) with a linear fast dynamics we end up with a reduced model that exhibits a structure for which we can design a circle criterion observer ${ }^{2}$.

Assumption 10. The matrix $M_{2}$ in $44 \mathrm{~b}$ is Hurwitz.

Assumption 11. The solutions of the system belong to a compact set. Moreover, the functions $\gamma(\cdot)$ and $\sigma(\cdot, \cdot)$ are locally Lipschitz, and $\gamma(\cdot)$ satisfies Assumption 1 in Chong et al ${ }^{4}$. 
Assumption 11 over $\sigma(\cdot, \cdot)$ is useful to prevent the solutions of $x$ from escaping to infinity in a finite time ${ }^{2}$. Note that this example satisfies our results relaying in Remark 12 since we consider a local Lipschitz condition. From Assumption 11 we know that for any $i$-entry $\left(\gamma_{i}\right)$ of the vector $\gamma$, there exists a time-varying gain $\delta_{i}(t)$ taking values in the interval $\left[0, L_{i}\right]$ such that

$$
\gamma_{i}\left(a_{i}\right)-\gamma_{i}\left(b_{i}\right) \leq \delta_{i}(t)\left(a_{i}-b_{i}\right), \forall a_{i}, b_{i} \in \mathbb{R},
$$

where $L_{i}$ is a Lipschitz constant for $\gamma_{i}$. This property must hold in order to implement the circle criterion observer introduced by Chong et $\mathrm{al}^{4}$. We now check our assumptions for the class of systems represented by (44). To obtain the lower dimensional systems, we set $\epsilon=0$ such that the system is restricted to the slow manifold

$$
M_{1} x+M_{2} z=0 .
$$

Then, it follows that, $H(x)=-M_{2}^{-1} M_{1} x$, is an isolated solution of 46 . Then, Assumption 1 holds with $H(x)=-M_{2}^{-1} M_{1} x$ which always exists by virtue of Assumption 10 . By using $H(x)$, we have that the reduced system is given by

$$
\begin{aligned}
\dot{x} & =A_{0} x+G \gamma(\bar{F} x)+\sigma\left(y_{s}, u\right), \\
y_{s} & =C x+D w,
\end{aligned}
$$

where $A_{0}=A-B M_{2}^{-1} M_{1}, C=C_{1}-C_{2} M_{2}^{-1} M_{1}$, and $\bar{F}=F_{1}-F_{2} M_{2}^{-1} M_{1}$. Note that it is assumed that the pair $\left(A_{0}, C\right)$ is detectable. Therefore, to allow more generality for the matrix $A_{0}$, we need to assume that the reduced system (47) is input-tostate practical stable (ISpS), such that there exists a Lyapunov ISpS function that satisfies Assumption 2 This is required since there is no need for $A_{0}$ to be Hurwitz.

Remark 16. If the matrix $A_{0}$ is Hurwitz, Assumption 2 holds with $V_{1}(x)$ being a quadratic Lyapunov function. Moreover, it is straightforward to find the functions and constants for which Assumption 4 is satisfied.

We now define the change of variables $z=\xi-M_{2}^{-1} M_{1} x$. Then, the original system 44 in the $(x, \xi)$ variables is given by

$$
\begin{aligned}
\dot{x} & =A x+G \gamma\left(F_{1} x+F_{2}\left(\xi-M_{2}^{-1} M_{1} x\right)\right)+\sigma(y, u)+B\left(\xi-M_{2}^{-1} M_{1} x\right), \\
\epsilon \dot{\xi} & =M_{2} \xi+\epsilon\left(M_{2}^{-1} M_{1}\right)\left[A x+G \gamma\left(F_{1} x+F_{2}\left(\xi-M_{2}^{-1} M_{1} x\right)\right)+\sigma(y, u)+B\left(\xi-M_{2}^{-1} M_{1} x\right)\right], \\
y & =C x+C_{2} \xi+D w
\end{aligned}
$$

By expressing (48) in the fast time-scale $\tau=t / \epsilon$, we have that the boundary layer system at $\epsilon=0$ is given by

$$
\frac{d \xi}{d \tau}=M_{2} \xi
$$

Since $M_{2}$ is Hurwitz, we have from Theorem 4.6 in Khalil ${ }^{29}$ that for any given positive definite symmetric matrix $Q_{\xi}$ there exists a positive definite symmetric matrix $P_{\xi}$ that satisfies the following Lyapunov equation

$$
P_{\xi} M_{2}+M_{2}^{T} P_{\xi}=-Q_{\xi}
$$

To check Assumption 3. consider $W(\xi)=\xi^{T} P_{\xi} \xi$ as a candidate Lyapunov function for 49 . It follows that

$$
\frac{\partial W}{\partial \xi} M_{2} \xi \leq-\lambda_{\min }\left\{Q_{\xi}\right\}|\xi|^{2},
$$

Therefore, Assumption 3 is satisfied with $\underline{\alpha}_{W}(|\xi|)=\lambda_{\min }\left\{P_{\xi}\right\}|\xi|^{2}$ and $\bar{\alpha}_{W}(|\xi|)=\lambda_{\max }\left\{P_{\xi}\right\}|\xi|^{2}$ as the lower and upper bounds for $W(\xi)$ respectively, and with $\zeta_{3}=\lambda_{\min }\left\{Q_{\xi}\right\}$ and $\alpha_{W}(|\xi|)=|\xi|$ as the terms satisfying [51). Due to the generality of the matrix $A_{0}$, we need to assume that the full system (48) satisfies the interconnection conditions in Assumption 4 We now consider the circle criterion observer proposed in Section 5.1 of $\mathrm{Arcak}^{[2}$ and in Chong et al ${ }^{[4}$ with the following dynamics

$$
\dot{\hat{x}}=A_{0} \hat{x}+L(C \hat{x}-y)+G \gamma(\bar{F} \hat{x}+K(C \hat{x}-y))+\sigma(y, u),
$$

where $\hat{x} \in \mathbb{R}^{n}$ is the observer's state and an estimate of the state, $K$ and $L$ are gain matrices of appropriate dimensions which must be designed. By following the approach described in this manuscript, the observer (52) is designed for the reduced system (47), and then implemented on the full singularly perturbed plant (48). Since we are dealing with a full order observer, it follows that Assumption 5 trivially holds because the output of the observer is a linear map in which the transformation matrix is the identity matrix. We now define the estimation error as $e:=x-\hat{x}$. It follows that the error dynamics are given by

$$
\dot{e}=\left(A_{0}+L C\right) e-L D w+G[\gamma(\bar{F} x)-\gamma(\bar{F}(x-e)-K C e-K D w)] .
$$


To check Assumption 6, we consider the Lyapunov function $V_{3}(e)=e^{T} P_{3} e$, where $P_{3}=P_{3}^{T}>0$. The matrix $P_{3}$ is obtained by solving the following LMI from Chong et al ${ }^{2}$

$$
\left[\begin{array}{ccc}
\left(A_{0}+L C\right)^{T} P_{3}+P_{3}\left(A_{0}+L C\right)+\hat{v} I & P_{3} G+(F+K C)^{T} \Lambda & -P_{3} L D \\
G^{T} P_{3}+\Lambda(F+K C) & -2 \Lambda\left(\frac{1}{L_{1}}, \cdots, \frac{1}{L_{n_{\gamma}}}\right) & -\Lambda K D \\
-P_{3} L D & -\Lambda K D & \mu_{w} I
\end{array}\right] \leq 0,
$$

where $\Lambda>0$ is a diagonal matrix and an observer design parameter, $\mu_{w}>0$ is a scalar constant and $\hat{v}>0$ is also an observer design parameter. When the LMI in $(54)$ is satisfied, it follows from Chong et al ${ }^{4}$ that

$$
\frac{\partial V_{3}}{\partial e} f_{e}(x, e) \leq-\hat{v}|e|^{2}+\mu_{w}|w|^{2},
$$

with $f_{e}(x, e)=\left(A_{0}+L C\right) e-L D w+G[\gamma(\bar{F} x)-\gamma(\bar{F}(x-e)-K C e-K D w)]$. Then, Assumption 6 holds with $\underline{\alpha}_{V_{3}}(|e|)=\lambda_{\min }\left\{P_{3}\right\}|e|^{2}$ and $\bar{\alpha}_{V_{3}}(|e|)=\lambda_{\max }\left\{P_{3}\right\}|e|^{2}$ being the lower and upper bounds for $V_{3}(|e|)$ respectively, with $\zeta_{2}=\hat{v}$, $\alpha_{V_{3}}(|e|)=|e|$ and $\gamma_{V_{3}}(|w|)=\mu_{w}|w|^{2}$ being the elements that satisfy the bound in $\sqrt[55]{ }$, and with $\hat{\zeta}_{2}=2\left|P_{3}\right|$ being the constant that multiplies $\alpha_{V_{3}}(\cdot)$ to bound the norm of the gradient of $V_{3}(e)$ with respect to $e$.

It follows from Remark 8 that Assumption 7 holds when using the Lyapunov function $V_{3}(\hat{x})$ as a Lyapunov function for the observer dynamics (52). Moreover, Assumption 8 holds globally since the output does not depend on the perturbation parameter. Then, Assumption 8 is satisfied with $\alpha_{y}(|(x, \xi)|)=2 \max \left\{|C|,\left|C_{2}\right|\right\}|(x, \xi)|$ and $\gamma_{w}(|w|)=|D||w|$. Note that in the case of full order observers we do not need Assumptions 5, 7 and 8 and Corollary 2 since the error dynamics completely capture the performance of the observer states.

To verify Assumption 9. we need to obtain the error dynamics when the observer (52) is implemented on the full system (48). Then, by considering the full system we obtain that the error dynamics are given as follows

$$
\dot{e}=\left(A_{0}+L C\right) e+G \gamma\left(\left[\bar{F}, F_{2}\right][x, \xi]^{T}\right)+B \xi+L C_{2} \xi-L D w-G \gamma\left(\bar{F}(x-e)-K\left(C e+C_{2} \xi\right)-K D w\right) .
$$

By considering Assumption 11 and equations (53) and (56), we have that Assumption 9 holds with $b_{6}=2\left(\left|P_{1}\right|\left|B+L C_{2}\right|+\right.$ $\left.L_{0}\left|P_{1} G\right|\left|F_{2}\right|+L_{0}\left|P_{1} G\right|\left|K C_{2}\right|\right)$, while the rest of the constants and functions are zero. $L_{0}$ is the Lipschitz constant on the compact set where the solutions belong. We have checked that Assumption 1-9 hold for plants in the form of (44) and the circle criterion observer (52). Therefore, we conclude that all our results holds relaying on Remark 12. We summarise this section in the following corollary which is an immediate consequence of Theorem 1 .

Corollary 4. Consider the singularly perturbed plant (44), the circle criterion observer $(52)$ and the error dynamics $(56)$. If Assumptions 2, 4,10 and 11 hold, there exists a positive definite matrix $P_{3}$, constants $\hat{v}>0$ and $\mu_{w}>0$, such that for any $\Delta>0, \Delta_{u_{1}}>0, \Delta_{u_{2}}>0, \Delta_{w}>0$ and $\mu>0$, there exists $T^{*}>0$ and $\epsilon^{*}>0$ such that

$$
|e(t)| \leq \sqrt{\frac{\lambda_{\max }\left(P_{3}\right)}{\lambda_{\min }\left(P_{3}\right)}}\left|e_{0}\right| \exp \left(-\frac{\hat{v}}{2 \lambda_{\max }\left(P_{3}\right)} t\right)+\sqrt{\frac{8 \lambda_{\max }\left(P_{3}\right)}{\hat{v} \lambda_{\min }\left(P_{3}\right)} \mu_{w}\left\|w\left[t_{0}, t\right]\right\|^{2}}+\mu,
$$

for all $\epsilon \in\left(0, \epsilon^{*}\right),\left|\left(x_{0}, \xi_{0}, e_{0}\right)\right| \leq \Delta,\|u\|_{\infty} \leq \Delta_{u_{1}},\|\dot{u}\|_{\infty} \leq \Delta_{u_{2}},\|w\|_{\infty} \leq \Delta_{w}$, and $t \geq \epsilon T^{*}+t_{0}$. Furthermore, for the given $\Delta>0, \Delta_{u_{1}}>0, \Delta_{u_{2}}>0, \Delta_{w}>0$ and $\mu>0$, there exists $T^{*}>0$ and $\epsilon_{\mathcal{L}_{2}}^{*}>0$ such that

$$
\int_{t_{0}}^{t}|e(\tau)|^{2} d \tau \leq \frac{2 \lambda_{\max }\left(P_{3}\right)}{\hat{v}}\left|e_{0}\right|+\frac{2 \mu_{w}}{\hat{v}} \int_{t_{0}}^{t}|w(\tau)|^{2} d \tau+\mu\left(t-t_{0}\right) .
$$

for all $\epsilon \in\left(0, \epsilon_{\mathcal{L}_{2}}^{*}\right),\left|\left(x_{0}, \xi_{0}, e_{0}\right)\right| \leq \Delta$, for any input satisfying $\|u\|_{\infty} \leq \Delta_{u_{1}},\|\dot{u}\|_{\infty} \leq \Delta_{u_{2}},\|u\|_{\mathcal{L}_{2}} \leq \Delta_{u_{1}},\|\dot{u}\|_{\mathcal{L}_{2}} \leq \Delta_{u_{2}}$, for any $\|w\|_{\infty} \leq \Delta_{w},\|w\|_{\mathcal{L}_{2}} \leq \Delta_{w}$ and for all $t \geq \epsilon T^{*}+t_{0}$.

\section{2 | Reduced-Order Circle Criterion Observer}

Reduced-order observers are useful in a number of applications in which it might be more convenient to estimate only the unmeasured states. Here, we deal with a reduced-order version of the circle criterion observer considered in Section 5.1 In this section, we follow the same approach as in the previous one to analyse the robustness, with respect singular perturbations, of the reduced-order circle criterion observer introduced in Section 5.2 of $\operatorname{Arcak}^{2}$. 
The class of plants we consider in this section is the same class of systems introduced in Section 5.1. but with an special output map. Then, we just make reference to the content in Section 5.1 and mention the slight differences considered for this new case. Here, we focus on analysing nonlinear singularly perturbed systems with an structure given by (44a)-(44b), and an output defined by

$$
y=E x
$$

where $E \in \mathbb{R}^{p \times n}$ is defined as $E:=\left[\begin{array}{ll}I & \mathbf{0}\end{array}\right]$ with $I$ being a $p \times p$ identity matrix and $\mathbf{0}$ being a $p \times(n-p)$ zero matrix. The definition of the output implies that $y$ consists of $p$ elements of the state vector. We consider Assumptions 10 and 11 for this case too. It is observed that Assumptions 1 - 4 are only related to the system dynamics without considering the output. Since we already analysed the plant in Section 5.1, we now focus on the reduced-order circle criterion observer introduced in Section 5.2 of $\operatorname{Arcak}^{[2}$ when is used to estimate the slow states of the system. To design a reduced-order observer for the slow system (47), we need an extra assumption over the model (47).

Assumption 12. There is a change of coordinates such that the slow state vector is given by $x=\left[y^{T}, x_{o}^{T}\right]^{T}$. Moreover, the slow system in the new coordinates is

$$
\begin{gathered}
\dot{y}=A_{1} x_{o}+G_{1} \gamma\left(\left[\bar{F}_{1}, \bar{F}_{2}\right]\left[y, x_{o}\right]^{T}\right)+\sigma_{1}(y, u), \\
\dot{x}_{o}=A_{2} x_{o}+G_{2} \gamma\left(\left[\bar{F}_{1}, \bar{F}_{2}\right]\left[y, x_{o}\right]^{T}\right)+\sigma_{2}(y, u),
\end{gathered}
$$

where the linear terms in $y$ are incorporated in the nonlinearities $\sigma_{1}(y, u)$, and $\sigma_{2}(y, u)$, and $\bar{F}_{1}$ and $\bar{F}_{2}$ are matrices of appropriate dimensions.

By following the design process given in Arcak ${ }^{2}$, we have that the estimate of the unmeasured variable $x_{o}$ is obtained via $\chi=x_{o}+N y$, where $N \in \mathbb{R}^{(n-p) \times p}$ is to be designed. The following auxiliary subsystem is constructed from the definition of $\chi$,

$$
\dot{\chi}=\left(A_{2}+N A_{1}\right) \chi+\left(G_{2}+N G_{1}\right) \gamma\left(\bar{F}_{2} \chi+\left(\bar{F}_{1}-\bar{F}_{2} N\right) y\right)+\tilde{\sigma}(y, u),
$$

where $\tilde{\sigma}(y, u)=N \sigma_{1}(y, u)+\sigma_{2}(y, u)-\left(A_{2}+N A_{1}\right) y$. The reduced-order observer is designed by considering the auxiliary system (61). Then, the observer dynamics are given by

$$
\dot{\hat{\chi}}=\left(A_{2}+N A_{1}\right) \hat{\chi}+\left(G_{2}+N G_{1}\right) \gamma\left(\bar{F}_{2} \hat{\chi}+\left(\bar{F}_{1}-\bar{F}_{2} N\right) y\right)+\tilde{\sigma}(y, u),
$$

The estimate of $x_{o}$, i.e., the output of the observer is given by $\hat{x}_{o}=\hat{\chi}-N y$, which agrees with our framework relaying on Remarks 5, 7 and 10. The estimation error is defined as $e=x_{o}-\hat{x}_{o}=\chi-\hat{\chi}$ which verify that this design method fits our theory since conditions in Remark 5 hold. It follows that the error dynamics are given by

$$
\dot{e}=\left(A_{2}+N A_{1}\right) e+\left(G_{2}+N G_{1}\right)\left[\gamma\left(\bar{F}_{2} \chi+\left(\bar{F}_{1}-\bar{F}_{2} N\right) y\right)-\gamma\left(\bar{F}_{2}(\chi-e)+\left(\bar{F}_{1}-\bar{F}_{2} N\right) y\right)\right] .
$$

It can be proven that $y$ can be removed from the error dynamics (63) such that it becomes a function that only depends on $e$, see Section 5.2 of $\operatorname{Arcak}^{2}$. The alternative representation of $(63)$ is

$$
\dot{e}=\left(A_{2}+N A_{1}\right) e+\left(G_{2}+N G_{1}\right) \psi\left(t, \bar{F}_{2} e\right),
$$

where $\psi(\cdot, \cdot)=\gamma\left(\bar{F}_{2} \chi+\left(\bar{F}_{1}-\bar{F}_{2} N\right) y\right)-\gamma\left(\bar{F}_{2}(\chi-e)+\left(\bar{F}_{1}-\bar{F}_{2} N\right) y\right)$. To verify Assumption 6 , we consider the Lyapunov function $V_{3}(e)=e^{T} \hat{P} e$, where $\hat{P}=\hat{P}^{T}>0$ is different from the the matrix considered in Section 5.1 Here, the matrix $\hat{P}$ is obtained by solving the following LMI

$$
\left[\begin{array}{cc}
\left(A_{2}+N A_{1}\right)^{T} \hat{P}+\hat{P}\left(A_{2}+N A_{1}\right)+\hat{v} I & \hat{P}\left(G_{2}+N G_{1}\right)+\bar{F}_{2}^{T} \Lambda \\
\left(G_{2}+N G_{1}\right)^{T} \hat{P}+\Lambda \bar{F}_{2} & 0
\end{array}\right] \leq 0,
$$

where $\Lambda>0$ is a diagonal matrix and an observer design parameter, and $\hat{v}>0$ is also an observer design parameter. It follows from $\operatorname{Arcak}^{2}$ that

$$
\frac{\partial V_{3}}{\partial e} f_{e}(e) \leq-\hat{v}|e|^{2}
$$

when the LMI 65$)$ is satisfied. Note that $f_{e}(e)=\left(A_{2}+N A_{1}\right) e+\left(G_{2}+N G_{1}\right) \psi\left(t, \bar{F}_{2} e\right)$ in $(66)$. Therefore, we conclude that Assumption 6 is satisfied with $\underline{\alpha}_{V_{3}}(|e|)=\lambda_{\min }\{\hat{P}\}|e|^{2}$ and $\bar{\alpha}_{V_{3}}(|e|)=\lambda_{\max }\{\hat{P}\}|e|^{2}, \zeta_{2}=\hat{v}, \alpha_{V_{3}}(|e|)=|e|$, and $\hat{\zeta}{ }_{2}=2|\hat{P}|$. We know from Remark 8 that it is not mandatory to check Assumption 7 when the reduced-order observer relies on Remark 5. For this case, Assumption 7 can be verified by using the Lyapunov function $V_{3}(\hat{\chi})$ as a Lyapunov function for the observer dynamics 62 . Moreover, Assumption 8 holds globally with $\left.\alpha_{y}(|(x, \xi)|)=2|E|\right\}|(x, \xi)|$ since the output does not depend on the 
perturbation parameter. Similar to the full-order observers case, we do not need Assumptions 5 , 7 and 8 and Corollary 2 since the error dynamics completely capture the performance of the observer states.

Under the proposed approach, the observer designed for the reduced model must be implemented in the full system. Then, we have that the error dynamics when the observer is applied to the true system are given by

$$
\dot{e}=\left(A_{2}+N A_{1}\right) e+B_{o} \xi+\left(G_{2}+N G_{1}\right)\left[\gamma\left(\bar{F}_{2} \chi+\left(\bar{F}_{1}-\bar{F}_{2} N\right) y+F_{2} \xi\right)-\gamma\left(\bar{F}_{2}(\chi-e)+\left(\bar{F}_{1}-\bar{F}_{2} N\right) y+F_{2} \xi\right)\right],
$$

where $B_{o}$ is a matrix of appropriate dimensions that agrees with the unmeasured state. Then, it follows that Assumption 9 is satisfied with $b_{6}=2|\hat{P}|\left|B_{0}\right|$, while the rest of the constants and functions of $u$ are zero. Since Assumption 1 - 9 hold, we conclude that our framework applies to the class of systems and the reduced-order observer considered in this section. We summarise the content of this section in the next corollary which is an immediate consequence of Theorem 1 and Corollary 3.

Corollary 5. Consider the singularly perturbed plant $(\sqrt{60})$, the circle criterion observer $(62)$ and the error dynamics $(67)$. If Assumptions 2, 4 and 10- 12 hold, there exists a positive definite matrix $\hat{P}$ and $\hat{v}>0$, such that for any $\Delta>0, \Delta_{u_{1}}>0, \Delta_{u_{2}}>0$ and $\mu>0$, there exists $T^{*}>0$ and $\epsilon^{*}>0$ such that

$$
|e(t)| \leq \sqrt{\frac{\lambda_{\text {max }}(\hat{P})}{\lambda_{\text {min }}(\hat{P})}}\left|e_{0}\right| \exp \left(-\frac{\hat{v}}{2 \lambda_{\text {max }}(\hat{P})} t\right)+\mu,
$$

for all $\epsilon \in\left(0, \epsilon^{*}\right),\left|\left(x_{0}, \xi_{0}, \chi_{0}, e_{0}\right)\right| \leq \Delta,\|u\|_{\infty} \leq \Delta_{u_{1}},\|\dot{u}\|_{\infty} \leq \Delta_{u_{2}}$, and $t \geq \epsilon T^{*}+t_{0}$. Furthermore, for the given $\Delta>0$, $\Delta_{u_{1}}>0, \Delta_{u_{2}}>0$ and $\mu>0$, there exists $T^{*}>0$ and $\epsilon_{\mathcal{L}_{2}}^{*}>0$ such that

$$
\int_{t_{0}}^{t}|e(\tau)|^{2} d \tau \leq \frac{2 \lambda_{\max }(\hat{P})}{\hat{v}}\left|e_{0}\right|+\mu\left(t-t_{0}\right) .
$$

for all $\epsilon \in\left(0, \epsilon_{\mathcal{L}_{2}}^{*}\right),\left|\left(x_{0}, \xi_{0}, \chi_{0}, e_{0}\right)\right| \leq \Delta$, for any input satisfying $\|u\|_{\infty} \leq \Delta_{u_{1}},\|\dot{u}\|_{\infty} \leq \Delta_{u_{2}},\|u\|_{\mathcal{L}_{2}} \leq \Delta_{u_{1}},\|\dot{u}\|_{\mathcal{L}_{2}} \leq \Delta_{u_{2}}$ and for all $t \geq \epsilon T^{*}+t_{0}$.

\section{3 | High-Gain Observer with Limited Gain Power}

We now analyse the class of singularly perturbed systems which has a structure such that the reduced (slow) model takes the form in which results from Astolfi and Marconi ${ }^{8}$ can be applied to design a higher-order observer. It is observed that this class of plants is covered by the general model 11 . Moreover, available results ${ }^{112224}$ do not cover the class of systems and the observer considered in this section. Consider the class of systems with the following form

$$
\begin{aligned}
\dot{\mathbf{x}} & =f_{s}(\mathbf{x}, \mathbf{z}), \\
\epsilon \dot{\mathbf{z}} & =f_{f}(\mathbf{x}, \mathbf{z}), \\
y & =h(\mathbf{x}, \mathbf{z})+w(t),
\end{aligned}
$$

where the state vector $\mathbf{x} \in \mathbf{X} \subset \mathbb{R}^{n}$ corresponds to the slow state, $\mathbf{z} \in \mathbf{Z} \subset \mathbb{R}^{m}$ is the fast state, $y \in \mathbb{R}^{p}$ is the measured output variable, $w \in \mathbb{R}$ is the measurement noise which belongs to $\mathcal{L}_{\infty}$ and $\mathcal{L}_{2}$, and $\epsilon$ is the perturbation parameter of the process. We now check our assumptions for the class of systems represented by $(70)$. To obtain the lower dimensional systems, we set $\epsilon=0$ such that the system 70 is restricted to the slow manifold $0=f_{f}(\mathbf{x}, \mathbf{z})$.

Assumption 13. The algebraic equation $0=f_{f}(\mathbf{x}, \mathbf{z})$ has a solution $H(\mathbf{x})$ which can be obtained analytically.

Then, Assumption 1 holds by virtue of Assumption 13 . Note that the reduced system is given by

$$
\begin{aligned}
\dot{\mathbf{x}} & =f_{s}(\mathbf{x}, H(\mathbf{x})), \\
y_{s} & =h(\mathbf{x}, H(\mathbf{x}))+w(t) .
\end{aligned}
$$


Assumption 14. The reduced system (71) is input-to-state practical stable, such that there exists a Lyapunov ISpS function that satisfies Assumption 2 Moreover, there exists a transformation $x=\phi_{x}(\mathbf{x})$, such that the reduced system (71) can be written as

$$
\begin{aligned}
& \dot{x}=A_{n} x+B_{n} \psi(x), \\
& y=C_{n} x+w(t),
\end{aligned}
$$

where $\psi(\cdot)$ is a locally Lipschitz function, and $\left(A_{n}, B_{n}, C_{n}\right)$ is a triplet in "prime form" of dimension $n$, that is

$$
A_{n}=\left(\begin{array}{cc}
0_{(n-1) \times 1} & I_{n-1} \\
0 & 0_{1 \times(n-1)}
\end{array}\right), \quad B_{n}=\left(\begin{array}{c}
0_{(n-1) \times 1} \\
1
\end{array}\right), \quad C_{n}=\left(\begin{array}{ll}
1 & 0_{1 \times(n-1)}
\end{array}\right) .
$$

The system $(72)$ is defined on the set $\mathbb{X} \subset \mathbb{R}^{n}$ where $\mathbb{X}=\phi_{x}(\mathbf{X})$.

Note that the ISpS condition in Assumption 14 can be checked either in (71) or (72). It follows that Assumption 2 holds by virtue of Assumption 14 We have that the boundary layer system is given by

$$
\frac{d \xi}{d \tau}=f_{f}(\mathbf{x}, \xi+H(\mathbf{x}))
$$

where $\tau=t / \epsilon$ is the fast-time scale and $\xi=\mathbf{z}-H(\mathbf{x})$.

Assumption 15. There is a Lyapunov function $W(\xi)$ such that it can be proven that the boundary layer system is asymptotically stable. Furthermore, the full system (70) satisfies the interconnection conditions in Assumption 4

We require Assumption 15 to hold due to the generality of the boundary layer system (73). It follows from Assumption 15 that Assumptions 3 and 4 are satisfied. We now consider the high-gain limited power observer ${ }^{[8}$ with the following dynamics

$$
\begin{aligned}
\dot{\chi}_{i} & =A \chi_{i}+N \chi_{i+1}+D_{2}(\ell) K_{i} \hat{e}_{i}, \quad i=1, \ldots, n-2, \\
& \vdots \\
\dot{\chi}_{n-1} & =A \chi_{n-1}+B \psi_{s}(\hat{x})+D_{2}(\ell) K_{(n-1)} \hat{e}_{n-1},
\end{aligned}
$$

where $(A, B, C)$ is a triplet in prime form of dimension $2, \chi=\operatorname{col}\left(\xi_{1}, \ldots, \xi_{n-1}\right) \in \mathbb{R}^{2 n-2}$ is the state of the observer with $\chi_{i} \in \mathbb{R}^{2}$, $K_{i}=\left(k_{i 1}, k_{i 2}\right)^{T}$ are the gains to be designed, $D_{2}(\ell)=\operatorname{diag}\left(\ell, \ell^{2}\right)$ with $\ell$ being the high gain parameter, $\hat{x}=L_{1} x$ is the output of the observer with $L_{1}=\operatorname{blkdiag}(\underbrace{C, \ldots, C}, I_{2}), \hat{e}_{1}=y-C \chi_{1}, \hat{e}_{i}=B^{T} \chi_{i-1}-C \chi_{i}(i=2, \ldots, n-1)$, and $\psi_{s}(\cdot)$ is an appropriate $(n-2)$ times

saturated version of $\psi(\cdot)$. Note that state of the observer has a dimension of $2 n-2$ so that the redundancy of the observer is used to obtain tow estimates with the asymptotic properties of the standard high-gain observer. Since the output of the observer is a linear map, it follows that Assumption 5 trivially holds. We now define the estimation error as $e:=\hat{x}-x$. It can be show that the error dynamics are given by

$$
\dot{e}=\ell L_{1} M L_{1}^{-} e+L_{1}\left[\ell^{-(n-1)}\left(B_{2 n-2} \Delta \psi_{\ell}(e, x)+w_{\ell}(t)\right)\right],
$$

where $L_{1}^{-}$is the left inverse of $L_{1}, \Delta \psi_{\ell}(\cdot, \cdot)=\psi_{s}(e+x)-\psi(x), w_{\ell}(\cdot)=\ell^{n} \bar{K}_{1} w(\cdot)$ with $\bar{K}_{1}=\operatorname{col}\left(K_{1}, 0, \ldots, 0\right)$, and

$$
M=\left(\begin{array}{ccccccc}
E_{1} & N & 0 & & \cdots & \cdots & 0 \\
Q_{2} & E_{2} & N & \ddots & & & \vdots \\
0 & \ddots & \ddots & \ddots & \ddots & & \vdots \\
\vdots & \ddots & Q_{i} & E_{i} & N & \ddots & \vdots \\
\vdots & & & \ddots & Q_{n-2} & E_{n-2} & N \\
0 & \cdots & \cdots & \cdots & 0 & Q_{n-1} & E_{n-1}
\end{array}\right),
$$

with $E_{i}=\left(\begin{array}{ll}-k_{i 1} & 1 \\ -k_{i 2} & 0\end{array}\right), Q_{i}=\left(\begin{array}{ll}0 & k_{i 1} \\ 0 & k_{i 2}\end{array}\right), N=\left(\begin{array}{ll}0 & 0 \\ 0 & 1\end{array}\right)$. Since the solutions evolve in a compact set and $\psi(\cdot)$ is locally Lipschitz, it follows that $\psi(\cdot)$ is uniformly Lipschitz in $\mathbf{X}$ and $\psi_{s}(\cdot)$ is bounded. Moreover, there exists $\delta_{1}>0$ and $\delta_{2}>0$ such that

$$
\left|\ell^{-(n-1)} \Delta \psi_{\ell}(e, x)\right| \leq \delta_{1}|e|, \quad \text { and } \quad\left|\ell^{-(n-1)} w_{\ell}(t)\right| \leq \delta_{2}|\ell w(t)|,
$$

for all $e \in \mathbb{R}^{n}, x \in \mathbf{X}$ and $\ell \geq 1$. Now, let $P_{e}=P_{e}^{T}$ be such that $P_{e} M_{L}+M_{L}^{T} P_{e}=-I$ where $M_{L}=L_{1} M L_{1}^{-}$and $I$ is the identity matrix. Consider $V_{3}(e)=e^{T} P e$ as a candidate Lyapunov function for 75 . It can be proven that the time derivative of 
the aforementioned Lyapunov function along the solutions of $(75)$ is bounded as follows

$$
\left.\dot{V}_{3}\right|_{75} \leq-\mathbf{a}_{1} \ell|e|^{2}+\mathbf{a}_{2}|w|,
$$

for all $\ell \geq 4 \delta_{1}|P|$ where $\mathbf{a}_{1}$ and $\mathbf{a}_{2}$ are positive constants. Therefore, Assumption 6 holds with $\underline{\alpha}_{V_{3}}(|e|)=\lambda_{\min }\left\{P_{e}\right\}$, $\bar{\alpha}_{V_{3}}(|e|)=\lambda_{\max }\left\{P_{e}\right\}, \zeta_{2}=\mathbf{a}_{1} \ell, \alpha_{V_{3}}(|e|)=|e|^{2}, \gamma_{V_{3}}(|w|)=\mathbf{a}_{2}|w|$, and $\hat{\zeta}_{2}=2\left|P_{e}\right|$. The Lyapunov analysis carried out in Astolfi and Marconi ${ }^{8}$ considers the auxiliary variables $\eta_{i}=\ell^{2-i} D_{2}(\ell)^{-1}\left(\chi_{i}-\operatorname{col}\left(x_{i}, x_{i+1}\right)\right)$ to construct a vector $\eta=\operatorname{col}\left(\eta_{1}, \ldots, \eta_{n_{1}}\right)$ which represent the estimation error of the two available estimates of $x$.

By using the Lyapunov function used in Astolfi and Marconi ${ }^{8}$, it can be proven that 27 in Assumption 7 holds with $\alpha_{o_{1}}\left(\left|\chi_{0}\right|\right)=c_{1} \exp \left(-c_{2} \ell t\right)\left|\chi_{0}\right|, \alpha_{o_{2}}\left(\|y\|_{\infty}\right)=c_{3}|| y \|_{\infty}$ and $\alpha_{o_{3}}\left(\|u\|_{\infty}\right)=0$ where $c_{1}=\sqrt{\lambda_{\max }\{P\} / \lambda_{\min }\{P\}}, c_{2}=d_{1} / 2 \lambda_{\max }\{P\}$, and $c_{3}=\sqrt{\lambda_{\max }\{P\} / \lambda_{\min }\{P\}} d_{2}$ with $d_{1}>0, d_{2}>0$ and $P=P^{T}$ satisfying $P M+M^{T} P=-I$. Since we use a definition of $\mathcal{L}_{2}$ equivalent to ISS, the condition $(28)$ can be concluded from the same analysis.

Note that the output of the system does not depend on the perturbation parameter. Then, Assumption 8 must hold globally. Due to the generality of the output map, we assume that Assumption 8 holds. The generality of the system 770 does not allow to obtain a unique solution for the constants and functions in Assumption 9 The best we can do is to guarantee that Assumption 9 holds with $b_{6}>0$ and $b_{7} \geq 0$ while $a_{4}=0, a_{5}=0, a_{6}=0, a_{7}=0, \gamma_{5}(\cdot)=0$ and $\gamma_{6}(\cdot)=0$.

Remark 17. Sharper conclusions on the bounds in Assumption 9 can be concluded if we restrict the class of systems in (70). For instance, one can consider a linear fast dynamics.

The following result is a consequence of a direct application of Theorem 1 to the class of systems and the higher-order observer considered in this section.

Corollary 6. Consider the singularly perturbed plant (70), the higher-order observer (74) and the error dynamics (75). If Assumptions 13- 15 hold, there exists a positive definite matrix $P_{e}$, and constants $\mathbf{a}_{1}>0$ and $\mathbf{a}_{2}>0$, such that for any $\Delta>0$, $\Delta_{w}>0$ and $\mu>0$, there exists $T^{*}>0$ and $\epsilon^{*}>0$ such that

$$
|e(t)| \leq \sqrt{\frac{\lambda_{\max }\left(P_{e}\right)}{\lambda_{\min }\left(P_{e}\right)}}\left|e_{0}\right| \exp \left(-\frac{\mathbf{a}_{1} \ell}{2 \lambda_{\max }\left(P_{e}\right)} t\right)+\sqrt{\frac{8 \lambda_{\max }\left(P_{e}\right)}{\mathbf{a}_{1} \ell \lambda_{\min }\left(P_{e}\right)} \mathbf{a}_{2}\left\|w\left[t_{0}, t\right]\right\|^{2}}+\mu,
$$

for all $\epsilon \in\left(0, \epsilon^{*}\right),\left|\left(x_{0}, \xi_{0}, \chi_{0}, e_{0}\right)\right| \leq \Delta,\|w\|_{\infty} \leq \Delta_{w}$, and $t \geq \epsilon T^{*}+t_{0}$. Furthermore, for the given $\Delta>0, \Delta_{w}>0$ and $\mu>0$, there exists $T^{*}>0$ and $\epsilon_{\mathcal{L}_{2}}^{*}>0$ such that

$$
\int_{t_{0}}^{t}|e(\tau)|^{2} d \tau \leq \frac{2 \lambda_{\max }\left(P_{e}\right)}{\mathbf{a}_{1} \ell}\left|e_{0}\right|+\frac{2 \mathbf{a}_{2}}{\mathbf{a}_{1} \ell} \int_{t_{0}}^{t}|w(\tau)|^{2} d \tau+\mu\left(t-t_{0}\right) .
$$

for all $\epsilon \in\left(0, \epsilon_{\mathcal{L}_{2}}^{*}\right),\left|\left(x_{0}, \xi_{0}, \chi, e_{0}\right)\right| \leq \Delta$, for any $\|w\|_{\infty} \leq \Delta_{w},\|w\|_{\mathcal{L}_{2}} \leq \Delta_{w}$ and for all $t \geq \epsilon T^{*}+t_{0}$.

\section{4 | Simulation Results: An automotive suspension system}

Here, we present simulations results to illustrate the applicability of our results. Consider the quarter-car model of automotive suspension in Chapter 11 of Khalil ${ }^{[29}$ with nonlinear hardening spring between the car body and the tire. Furthermore, assume that there is no disturbance to the system. Let define $\dot{\mathbf{x}}=d \mathbf{x} / d t_{r}$ with $t_{r}=t \sqrt{k_{s} / m_{s}}$. Then, the model of the system can be expressed in the following standard singularly perturbed form 29

$$
\begin{aligned}
\dot{x}_{1} & =x_{2}-z_{2}, \\
\dot{x}_{2} & =-x_{1}-x_{1}^{3}-\beta\left(x_{2}-z_{2}\right)+u, \\
\epsilon \dot{z}_{1} & =z_{2}, \\
\epsilon \dot{z}_{2} & =\alpha x_{1}+\alpha x_{1}^{3}-\alpha \beta\left(z_{2}-x_{2}\right)-z_{1}-\alpha u,
\end{aligned}
$$

with $\epsilon=\sqrt{\frac{k_{s} m_{u}}{k_{t} m_{s}}}, \alpha=\sqrt{\frac{k_{s} m_{s}}{k_{t} m_{u}}}, \beta=\frac{b_{s}}{\sqrt{k_{s} m_{s}}}, u=\frac{F}{k_{s} \ell}$, where $m_{s}$ and $m_{u}$ are the car body and tire masses, $k_{s}$ and $k_{t}$ are the spring constants of the strut and tire, $\ell$ is a constant distance, which is used to normalize variables, $b_{s}$ is the shock absorber constant (damping term), and $F$ is a bounded force generated by a force generator that acts as an active element. By following the singular perturbation techniques, Assumption 1 holds with $H(x, u)=\left[\alpha x_{1}+\alpha x_{1}^{3}+\alpha \beta x_{2}-\alpha u, 0\right]^{T}$. Then, the reduced system system is 
given by

$$
\begin{aligned}
& \dot{x}_{1}=x_{2}, \\
& \dot{x}_{2}=-x_{1}-x_{1}^{3}-\beta x_{2}+u .
\end{aligned}
$$

While the boundary layer system in the fast time scale $\tau-t / \epsilon$, is defined by

$$
\begin{aligned}
& \frac{d \xi_{1}}{d \tau}=\xi_{2}, \\
& \frac{d \xi_{2}}{d \tau}=-\alpha \beta \xi_{2}-\xi_{1} .
\end{aligned}
$$

We now assume that an accelerometer, located on the car body, is used to measure the vertical velocity of the system; such a sensor allows having an output for the system in the form of

$$
y=x_{2}+w(t)
$$

where $w(t)=0.05 \sin (0.3 t)$ is the measurement noise. Note that the reduced system 80 with the output 82 can be written in the form of 477 with $\sigma(y, u)=[y, u-\beta y]^{T}$, and $A=\left[\begin{array}{cc}0 & 0 \\ -1 & 0\end{array}\right], G=\left[\begin{array}{c}0 \\ -1\end{array}\right], H=\left[\begin{array}{ll}1 & 0\end{array}\right], C=\left[\begin{array}{ll}0 & 1\end{array}\right], D=1$. Therefore, it follows that a circle-criterion observer (52) can be used to estimate the slow states of (79). Note that this problem falls within the framework presented in Section 5.1] Hence, the estimation error is expected to converge to a region around the origin as highlighted in Corollary 4. The aforementioned region is critically related to $\epsilon$ and to the bound of the measurement noise.

To perform simulations, we consider the following parameters: $k_{s}=500[N-m], k_{t}=6[K N-m], m_{s}=200[K g]$, $m_{u}=20[K g], b_{s}=35[N-s / m]$. For these values, we have that $\alpha=0.913$ and $\beta=0.111$. Note that, for the given parameters, the perturbation parameter for the system is $\epsilon=0.0913$. By following the design procedure described in Section 5.1. we obtain the following gain matrices for the circle-criterion observer $K=2.548, L=\left[\begin{array}{c}1.503 \\ -1.281\end{array}\right]$. It can be calculated from the values of the constants above and the Lyapunov functions $V_{1}(x)=\frac{1}{2}\left(x_{1}+\frac{1}{\beta} x_{2}\right)^{2}+\frac{1}{2}\left(1+\frac{1}{\beta^{2}}\right) x_{1}^{2}+\frac{1}{2} x_{2}^{2}$ and $W(\xi)=\left(\frac{\alpha \beta}{2}+\frac{1}{\alpha \beta}\right) \xi_{1}^{2}+$ $\xi_{1} \xi_{2}+\frac{1}{\alpha \beta} \xi_{2}^{2}$ that, for this example, Corollary 4 holds for all $\epsilon \in(0,0.129)$.

The performance of the Circle Criterion Observer designed for the slow system (80) and implemented on the full system (79) is presented in Figure 1 for different values of $\epsilon$. It is observed that the estimation error performs as expected, i.e. it converges to a small offset around the origin. Even though the Circle Criterion Observer has an exponential convergence rate for the reduced system, its performance on the original system is affected by the perturbation parameter and the fast part of the state as stated in our main result and illustrated in Figure 1
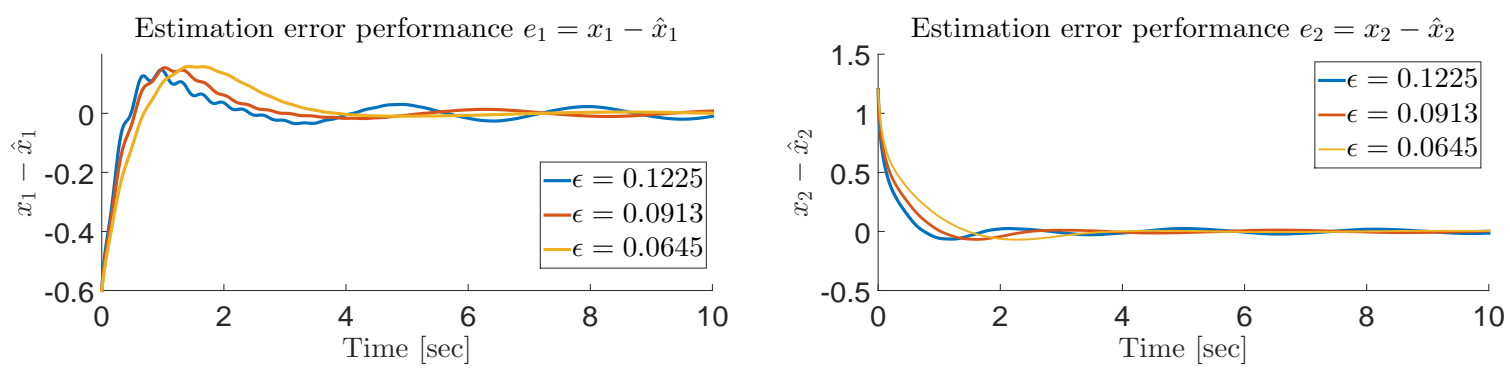

FIGURE 1 Estimation error performance for the estimates of $x_{1}$ and $x_{2}$ trough the Circle Criterion Observer.

\section{6 | CONCLUSIONS}

We have developed a new estimation framework for a general class of singularly perturbed systems in the standard form by considering nonlinear observers of general dimension. Estimation of the slow variables was analysed. Under the considered 
approach, an observer for the slow part of the system must be designed while the fast dynamics are neglected. The given conditions guarantee an acceptable performance of the error dynamics when the observer is implemented on the full system. We delivered results that show robustness of observers with respect to singular perturbations and with respect to measurement noise. The main contribution of this work fills in a gap in the literature. With this work, we cover a wide range of plants and nonlinear observers that were not covered by previous results. Moreover, the inclusion of the measurement noise gives significance to this work. Our results are useful to mathematically justify the observer design for the slow states by just considering the reduced (slow) model. We showed that three types of existing observers are covered by our results; therefore, we have illustrated and demonstrated the generality of our findings.

\section{APPENDIX}

\section{A . PROOF OF THEOREM 1}

We first prove that the error dynamics are ISS with respect to $x, \xi, u$ and $w$. Moreover, we show the system (30) satisfies a $\mathcal{L}_{2}$ stability property. These results are the key ingredients that allow us to show our main result in Section 4.1

Lemma 2. Consider the singularly perturbed system (30). If Assumptions 1 - 9 hold, there exists $\beta_{e}(\cdot, \cdot) \in \mathcal{K} \mathcal{L}$, functions $\gamma_{\xi}(\cdot), \gamma_{w}(\cdot) \in \mathcal{K}_{\infty}$, and class- $\mathcal{K}_{\infty}$ functions $\gamma_{x, \epsilon}(\cdot), \gamma_{u, \epsilon}(\cdot)$ parametrized by $\epsilon$ (their argument is of order $O(\epsilon)$ ), such that for any $\Delta_{L}>0, \Delta_{L_{u_{1}}}>0, \Delta_{L_{u_{2}}}>0$, and $\Delta_{L_{w}}>0$, there exists $\epsilon_{L}^{*}>0$, such that

$$
|e(t)| \leq \beta_{e}\left(\left|e_{0}\right|, t-t_{0}\right)+\gamma_{\xi}\left(\left\|\xi\left[t_{0}, t\right]\right\|\right)+\gamma_{x, \epsilon}\left(\left\|x\left[t_{0}, t\right]\right\|\right)+\gamma_{u, \epsilon}\left(\left\|u\left[t_{0}, t\right]\right\|\right)+\gamma_{w}\left(\left\|w\left[t_{0}, t\right]\right\|\right),
$$

for all $\epsilon \in\left(0, \epsilon_{L}^{*}\right)$ and for all $\left|\left(x_{0}, \xi_{0}, \chi_{0}, e_{0}\right)\right| \leq \Delta_{L},\|u\|_{\infty} \leq \Delta_{L_{u_{1}}},\|\dot{u}\|_{\infty} \leq \Delta_{L_{u_{2}}},\|w\|_{\infty} \leq \Delta_{L_{w}}$ and $t \geq t_{0} \geq 0$. Furthermore, there exists $k_{1}>0$ and $k_{i} \geq 0(i=2,3)$ such that

$$
\begin{aligned}
\int_{t_{0}}^{t} \alpha_{V_{3}}^{2}(|e(\tau)|) d \tau & \leq k_{1} \bar{\alpha}_{V_{3}}\left(\left|e_{0}\right|\right)+\epsilon k_{2} \int_{t_{0}}^{t} \alpha_{V_{1}}^{2}(|x(\tau)|) d \tau+\epsilon k_{1} \int_{t_{0}}^{t}\left[\gamma_{5}^{2}(|u(\tau)|)+\gamma_{6}^{2}(|u(\tau)|)\right] d \tau \\
& +k_{3} \int_{t_{0}}^{t} \alpha_{W}^{2}(|\xi(\tau)|) d \tau+k_{1} \int_{t_{0}}^{t} \gamma_{V_{3}}(|w(\tau)|) d \tau
\end{aligned}
$$

for all $\epsilon \in\left(0, \epsilon_{L}^{*}\right),\left|\left(x_{0}, \xi_{0}, \chi_{0}, e_{0}\right)\right| \leq \Delta_{L}$, for any input satisfying $\|u\|_{\infty} \leq \Delta_{L_{u_{1}}},\|\dot{u}\|_{\infty} \leq \Delta_{L_{u_{2}}},\|u\|_{\mathcal{L}_{2}} \leq \Delta_{L_{u_{1}}},\|\dot{u}\|_{\mathcal{L}_{2}} \leq \Delta_{L_{u_{2}}}$, for any $\|w\|_{\infty} \leq \Delta_{L_{w}},\|w\|_{\mathcal{L}_{2}} \leq \Delta_{L_{w}}$ and for all $t \geq t_{0} \geq 0$.

Proof of Lemma 2. We split the proof in two steps. In the first step, we prove that $\mathrm{A1}$ holds under Assumptions 1 - 9 . We then show that the error dynamics satisfy (A2).

Step 1) Let Assumptions 1 - 9 hold. Let $\lambda_{e}(\cdot, \cdot) \in \mathcal{K} \mathcal{L}$ be defined as the solution of the following scalar differential equation,

$$
\dot{y}_{e}=-\hat{\alpha}_{V_{3}}\left(y_{e}\right), \quad y_{e}\left(t_{0}\right)=y_{e_{0}},
$$

with $\hat{\alpha}_{V_{3}}(\cdot)=\frac{1}{4} \zeta_{2} \alpha_{V_{3}}^{2} \circ \bar{\alpha}_{V_{3}}^{-1}(\cdot)$ where $\zeta_{2}, \alpha_{V_{3}}(\cdot)$ and $\bar{\alpha}_{V_{3}}(\cdot)$ come from Assumption 6 . Then, $y(t)=\lambda_{e}\left(y_{e_{0}}, t-t_{0}\right)$. The existence of $\lambda_{e}(\cdot, \cdot)$ follows from Lemma 4.4 in Khalil ${ }^{29}$. Define the class- $\mathcal{K} \mathcal{L}$ function

$$
\beta_{e}(r, s):=\underline{\alpha}_{V_{3}}^{-1}\left(\lambda_{e}\left(\bar{\alpha}_{V_{3}}(r), s\right)\right),
$$


where the functions $\underline{\alpha}_{V_{3}}(\cdot)$ and $\bar{\alpha}_{V_{3}}(\cdot)$ come from Assumption 6 Define

$$
\begin{aligned}
\gamma_{\xi}(s) & :=\underline{\alpha}_{V_{3}}^{-1} \circ \bar{\alpha}_{V_{3}} \circ \tilde{\alpha}_{V_{3}}^{-1}\left(\frac{8\left(b_{4}+b_{5}+b_{6}+L b_{7}\right)^{2}}{\zeta_{2}^{2}} \alpha_{W}^{2}(s)\right), \\
\gamma_{x, \epsilon}(s) & :=\underline{\alpha}_{V_{3}}^{-1} \circ \bar{\alpha}_{V_{3}} \circ \tilde{\alpha}_{V_{3}}^{-1}\left(\epsilon \frac{8\left(a_{4}+a_{5}+a_{6}+L a_{7}\right)}{\zeta_{2}} \alpha_{V_{1}}^{2}(s)\right), \\
\gamma_{u, \epsilon}(s) & :=\underline{\alpha}_{V_{3}}^{-1} \circ \bar{\alpha}_{V_{3}} \circ \tilde{\alpha}_{V_{3}}^{-1}\left(\epsilon \frac{8}{\zeta_{2}}\left[\gamma_{5}^{2}(s)+\gamma_{6}^{2}(s)\right]\right), \\
\gamma_{w}(s) & :=\underline{\alpha}_{V_{3}}^{-1} \circ \bar{\alpha}_{V_{3}} \circ \tilde{\alpha}_{V_{3}}^{-1}\left(\frac{8}{\zeta_{2}} \gamma_{V_{3}}(s)\right) .
\end{aligned}
$$

where $\tilde{\alpha}_{V_{3}}(\cdot)=\alpha_{V_{3}}^{2}(\cdot)$, and all constants and functions come from Assumptions 2 - 5 . 8 and 9 , and $L>0$ is such that $\left|\partial h_{o} / \partial \chi\right| \leq L$ for all $\chi \in B_{1}$ with $B_{1}=\left\{\chi \in \mathbb{R}^{q}|| \chi \mid \leq \Delta_{1}\right\}$ where $\Delta_{1}>0$. Let $\Delta_{L}>0, \Delta_{L_{u_{1}}}>0, \Delta_{L_{u_{2}}}>0$, and $\Delta_{L_{w}}>0$ be given such that $\left|\left(x_{0}, \xi_{0}, \chi_{0}, e_{0}\right)\right| \leq \Delta_{L},\|u\|_{\infty} \leq \Delta_{L_{u_{1}}},\|\dot{u}\|_{\infty} \leq \Delta_{L_{u_{2}}}$, and $\|w\|_{\infty} \leq \Delta_{L_{w}}$. By using Lemma 1, we generate $\tilde{\epsilon}^{*}>0$ such that 177 holds for all $\epsilon \in\left(0, \tilde{\epsilon}^{*}\right)$. Define $\left(\frac{1}{\Delta}, \bar{\Delta}_{u_{1}}, \bar{\Delta}_{u_{2}}, \bar{\Delta}_{w}\right)$ as $\bar{\Delta}:=\Delta_{L}, \bar{\Delta}_{u_{1}}:=\Delta_{L_{u_{1}}}, \bar{\Delta}_{u_{2}}:=\Delta_{L_{u_{2}}}, \bar{\Delta}_{w}:=\Delta_{L_{w}}$. Using Corollary 2 , let $\left(\bar{\Delta}, \bar{\Delta}_{u_{1}}, \bar{\Delta}_{u_{2}}, \bar{\Delta}_{w}\right)$ generate $\hat{\epsilon}^{*}>0$ and $\Upsilon>0$ such that 32 holds for all $\epsilon \in\left(0, \hat{\epsilon}^{*}\right)$.

We now introduce $\Delta_{x}:=\beta_{L_{1}}\left(\Delta_{L}, 0\right)+\gamma_{L_{1}}\left(\Delta_{L_{u_{1}}}\right)+\tilde{\gamma}_{L_{1}}\left(\Delta_{L_{u_{1}}}\right)+\hat{\gamma}_{L_{1}}\left(\Delta_{L_{u_{2}}}\right)+\mu_{L_{1}}$ and $\Delta_{\chi}:=\alpha_{c_{1}}(\bar{\Delta})+\Upsilon$, where $\beta_{L_{1}}(\cdot, \cdot), \gamma_{L_{1}}(\cdot)$, $\tilde{\gamma}_{L_{1}}(\cdot), \hat{\gamma}_{L_{1}}(\cdot)$ and $\mu_{L_{1}}$ come from (17) in Lemma 1 , and $\alpha_{c_{1}}(\cdot)$ and $\Upsilon$ come from (32) in Corollary 2. Then, we have that $|x(t)| \leq \Delta_{x}$ for all $\left|\left(x_{0}, \xi_{0}\right)\right| \leq \Delta_{L}, u \in B_{u_{1}}, \dot{u} \in B_{u_{2}}$, and $t \geq t_{0} \geq 0$ where $B_{u_{1}}=\left\{u \in \mathbb{R}^{r}|| u \mid \leq \Delta_{L_{u_{1}}}\right\}$ and $B_{u_{2}}=\left\{\dot{u} \in \mathbb{R}^{r}|| \dot{u} \mid \leq \Delta_{L_{u_{2}}}\right\}$. From Lemma 1, we have that $|(x(t), \xi(t))| \leq \Delta_{x}$ for all $\left|\left(x_{0}, \xi_{0}\right)\right| \leq \Delta_{L}, u \in B_{u_{1}}, \dot{u} \in B_{u_{2}}$, and $t \geq t_{0} \geq 0$. Moreover, from the choice of $\left(\bar{\Delta}, \bar{\Delta}_{u_{1}}, \bar{\Delta}_{u_{2}}, \bar{\Delta}_{w}\right)$, we conclude that $|\chi(t)| \leq \Delta_{\chi}$ for all $\left|\left(x_{0}, \xi_{0}, \chi_{0}\right)\right| \leq \bar{\Delta}, u \in B_{u_{1}}, \dot{u} \in B_{u_{2}}$, and $t \geq t_{0} \geq 0$. Let $\epsilon_{L_{3}}^{*}>0$ be such that $\epsilon\left(a_{4}+a_{5}+a_{6}+L a_{7}+2\right)-\zeta_{2}<0$ for all $\epsilon \in\left(0, \epsilon_{L_{3}}^{*}\right), \epsilon_{L_{3}}^{*}$ is given below. Hence, define

$$
\epsilon_{L}^{*}:=\min \left\{\epsilon_{L_{1}}^{*}, \epsilon_{L_{2}}^{*}, \epsilon_{L_{3}}^{*}\right\},
$$

with

$$
\begin{aligned}
\epsilon_{L_{1}}^{*} & :=\frac{\frac{2}{3} \zeta_{1} \zeta_{3}}{b_{1}\left(b_{2}+b_{3}\right)+\zeta_{1}\left(a_{2}+a_{3}\right)+\frac{2}{3} \zeta_{3}\left(a_{1}+\frac{1}{4}\right)}, \\
\epsilon_{L_{2}}^{*}: & :=\epsilon_{y}, \\
\epsilon_{L_{3}}^{*} & :=\frac{\zeta_{2}}{a_{4}+a_{5}+a_{6}+L a_{7}+2},
\end{aligned}
$$

where all of the above constants come from Assumptions 2- 5,8 and 9

Note that A7a) comes from $\tilde{\epsilon}^{*}$ in Lemma 1. Moreover, from the choice of $\left(\bar{\Delta}, \bar{\Delta}_{u_{1}}, \bar{\Delta}_{u_{2}}, \bar{\Delta}_{w}\right)$ and Corollary 2 we have that A7b comes from $\hat{\epsilon}^{*}$. We have introduced above the condition from which $\epsilon_{L_{3}}^{*}$ has been constructed. To prove that (A1) holds, we now consider the Lyapunov function $V_{3}(t, e, x, \chi)$ in Assumption 6 and take its derivative along the solutions of (30), which is given by

$$
\left.\dot{V}_{3}\right|_{[30]}=\frac{\partial V_{3}}{\partial t}+\frac{\partial V_{3}}{\partial e} f_{e}(t, x, \chi, e, \xi+H(t, x, u), y, u, \dot{u}, \epsilon)+\frac{\partial V_{3}}{\partial x} f_{s}(t, x, \xi+H(t, x, u), u, \epsilon)+\frac{\partial V_{3}}{\partial \chi} f_{o}(t, \chi, y, u) .
$$

By adding and subtracting terms and using the definition of the error dynamics, we can rewrite $(\mathrm{A} 8)$ as follows

$$
\begin{aligned}
\left.\dot{V}_{3}\right|_{\text {30 }} & =\frac{\partial V_{3}}{\partial t}+\frac{\partial V_{3}}{\partial e} f_{e}\left(t, x, \chi, e, \xi+H(t, x, u), y_{s}, u, \dot{u}, 0\right)+\frac{\partial V_{3}}{\partial x} f_{s}(t, x, H(t, x, u), u, 0)+\frac{\partial V_{3}}{\partial \chi} f_{o}\left(t, \chi, y_{s}, u\right) \\
& +\frac{\partial V_{3}}{\partial x}\left[f_{s}(t, x, \xi+H(t, x, u), u, \epsilon)-f_{s}(t, x, H(t, x, u), u, 0)\right]+\frac{\partial V_{3}}{\partial \chi}\left[f_{o}(t, \chi, y, u)-f_{o}\left(t, \chi, y_{s}, u\right)\right] \\
& +\frac{\partial V_{3}}{\partial e}\left[f_{s}(t, x, H(t, x, u), u, 0)-f_{s}(t, x, \xi+H(t, x, u), u, \epsilon)\right]+\frac{\partial V_{3}}{\partial e}\left[\frac{\partial h_{o}}{\partial \chi}\left[f_{o}(t, \chi, y, u)-f_{o}\left(t, \chi, y_{s}, u\right)\right]\right] .
\end{aligned}
$$

As showed above, it follows from Corollary 2 that $|\chi| \leq \Delta_{\chi}$ for all $\epsilon \in\left(0, \hat{\epsilon}^{*}\right)$ where $\hat{\epsilon}^{*} \leq \epsilon_{L}^{*}$. Let define $\Delta_{1}:=\Delta_{\chi}$ and $\Delta_{2}:=\Delta_{L_{u_{1}}}$, so we have from Remark 4 that for the given $\Delta_{1}$ and $\Delta_{2}$ there is $L>0$ such that $\left|\partial h_{o} / \partial \chi\right| \leq L$ for all $\chi \in B_{1}$ 
with $B_{1}=\left\{\chi \in \mathbb{R}^{q}|| \chi \mid \leq \Delta_{1}\right\}$ where $\Delta_{1}:=\Delta_{\chi}$. By using the norm and applying inequalities in Assumptions 6 and 9 we have

$$
\begin{aligned}
\left.\dot{V}_{3}\right|_{\text {[30 }} & \leq-\zeta_{2} \alpha_{V_{3}}^{2}(|e|)+\epsilon\left(a_{4}+a_{5}+a_{6}+L a_{7}\right) \alpha_{V_{1}}(|x|) \alpha_{V_{3}}(|e|)+\left(b_{4}+b_{5}+b_{6}+L b_{7}\right) \alpha_{V_{3}}(|e|) \alpha_{W}(|\xi|) \\
& +\epsilon \gamma_{5}(|u|) \alpha_{V_{3}}(|e|)+\epsilon \gamma_{6}(|u|) \alpha_{V_{3}}(|e|)+\gamma_{V_{3}}(|w|) .
\end{aligned}
$$

Applying completion of squares to $\mathrm{A10}$ leads to

$$
\begin{aligned}
\left.\dot{V}_{3}\right|_{\text {[30 }} & \leq-\frac{3}{4} \zeta_{2} \alpha_{V_{3}}^{2}(|e|)+\epsilon \frac{1}{4}\left(a_{4}+a_{5}+a_{6}+L a_{7}+2\right) \alpha_{V_{3}}^{2}(|e|)+\epsilon\left(a_{4}+a_{5}+a_{6}+L a_{7}\right) \alpha_{V_{1}}^{2}(|x|) \\
& +\epsilon \gamma_{5}^{2}(|u|)+\epsilon \gamma_{6}^{2}(|u|)+\frac{\bar{k}_{1}}{\zeta_{2}} \alpha_{W}^{2}(|\xi|)+\gamma_{V_{3}}(|w|),
\end{aligned}
$$

with $\bar{k}_{1}=\left(b_{4}+b_{5}+b_{6}+L b_{7}\right)^{2}$. It follows from A11) that

$$
\left.\dot{V}_{3}\right|_{\text {[30 }} \leq-\frac{1}{2} \zeta_{2} \alpha_{V_{3}}^{2}(|e|)+\epsilon\left(a_{4}+a_{5}+a_{6}+L a_{7}\right) \alpha_{V_{1}}^{2}(|x|)+\epsilon \gamma_{5}^{2}(|u|)+\epsilon \gamma_{6}^{2}(|u|)+\frac{\bar{k}_{1}}{\zeta_{2}} \alpha_{W}^{2}(|\xi|)+\gamma_{V_{3}}(|w|),
$$

for all $\epsilon \in\left(0, \epsilon_{L_{3}}^{*}\right)$ with $\epsilon_{L_{3}}^{*}$ given by (A7c). It is observed that $\epsilon_{L_{3}}^{*} \leq \epsilon_{L}^{*}$. Then, it follows from (24) and (A12) that

$$
|e| \geq\left.\tilde{\alpha}_{V_{3}}^{-1}\left(\frac{4}{\zeta_{2}}\left[\epsilon\left(a_{4}+a_{5}+a_{6}+L a_{7}\right) \alpha_{V_{1}}^{2}(|x|)+\epsilon \gamma_{5}^{2}(|u|)+\epsilon \gamma_{6}^{2}(|u|)+\frac{\bar{k}_{1}}{\zeta_{2}} \alpha_{W}^{2}(|\xi|)+\gamma_{V_{3}}(|w|)\right]\right) \Rightarrow \dot{V}_{3}\right|_{[30,} \leq-\hat{\alpha}_{V_{3}}\left(V_{3}\right)
$$

where $\tilde{\alpha}_{V_{3}}(\cdot)=\alpha_{V_{3}}^{2}(\cdot)$ and $\hat{\alpha}_{V_{3}}(\cdot)=\frac{1}{4} \zeta_{2} \alpha_{V_{3}}^{2} \circ \bar{\alpha}_{V_{3}}^{-1}(\cdot)$. We can conclude an ISS result from A13 if the following condition holds

$$
\tilde{\alpha}_{V_{3}}^{-1}\left(\frac{4}{\zeta_{2}}\left[\epsilon\left(a_{4}+a_{5}+a_{6}+L a_{7}\right) \alpha_{V_{1}}^{2}\left(\Delta_{x}\right)+\epsilon \gamma_{5}^{2}\left(\Delta_{L_{u_{1}}}\right)+\epsilon \gamma_{6}^{2}\left(\Delta_{L_{u_{1}}}\right)+\frac{\bar{k}_{1}}{\zeta_{2}} \alpha_{W}^{2}\left(\Delta_{x}\right)+\gamma_{V_{3}}\left(\Delta_{L_{w}}\right)\right]\right) \leq \Delta_{L},
$$

for any $(x, \xi, \chi, e) \in B_{\rho}$, where $B_{\rho}:=\left\{(x, \xi, \chi, e) \in \mathbb{R}^{n} \times \mathbb{R}^{m} \times \mathbb{R}^{q} \times \mathbb{R}^{n}||(x, \xi, \chi, e) \mid \leq \underline{\alpha}^{-1} \circ \bar{\alpha}\left(\Delta_{L}\right)\right\}$. If A14 does not hold, the solutions would not belong to the invariant set that agrees with (24) and the dissipation inequality (A12), see Theorem 4.18 in Khali1 29 . We now exploit the cascade properties of the error dynamics, which are in cascade with the $x, \xi$ and $\chi$. Since Lemma 1 and Corollary 2 hold, $|x(t)| \leq|(x(t), \xi(t))|$ and $|\xi(t)| \leq|(x(t), \xi(t))|$, it follows that $x, \xi$ and $\chi$ are essentially bounded inputs to the error dynamics. Then, A13) implies that the error dynamics are ISS with respect to $x, \xi$, and $w$. By applying results in Sontag 26 and Sontag and Wang 27 , we obtain

$$
\begin{aligned}
|e(t)| \leq & \beta_{e}\left(\left|e_{0}\right|, t-t_{0}\right)+\underline{\alpha}_{V_{3}}^{-1} \circ \bar{\alpha}_{V_{3}} \circ \tilde{\alpha}_{V_{3}}^{-1}\left(\frac { 4 } { \zeta _ { 2 } } \left[\epsilon\left(a_{4}+a_{5}+a_{6}+L a_{7}\right) \alpha_{V_{1}}^{2}\left(|| x\left[t_{0}, t\right] \|\right)\right.\right. \\
& \left.\left.+\epsilon \gamma_{5}^{2}\left(\| u\left[t_{0}, t\right]||\right)+\epsilon \gamma_{6}^{2}\left(\| u\left[t_{0}, t\right]||\right)+\frac{\bar{k}_{1}}{\zeta_{2}} \alpha_{W}^{2}\left(\| \xi\left[t_{0}, t\right]||\right)+\gamma_{V_{3}}\left(\left\|w\left[t_{0}, t\right]\right\|\right)\right]\right),
\end{aligned}
$$

where $\beta_{e}(\cdot, \cdot) \in \mathcal{K} \mathcal{L}$ is given by [A4). By applying the weak triangle inequality to the second term on the right-hand side of A15, we conclude that A1p holds for all $\epsilon \in\left(0, \epsilon_{L}^{*}\right)$ and for all $\left|\left(x_{0}, \xi_{0}, \chi_{0}, e_{0}\right)\right| \leq \Delta_{L},\|u\|_{\infty} \leq \Delta_{L_{u_{1}}},\|\dot{u}\|_{\infty} \leq \Delta_{L_{u_{2}}},\|w\|_{\infty} \leq \Delta_{L_{w}}$ and $t \geq t_{0} \geq 0$, where $\gamma_{\xi}(\cdot), \gamma_{x, \epsilon}(\cdot), \gamma_{u, \epsilon}(\cdot)$, and $\gamma_{w}(\cdot)$ are given by A5 .

Step 2) We now prove that $(A 2)$ holds. Define

$$
\begin{aligned}
k_{1} & :=\frac{2}{\zeta_{2}}, \\
k_{2} & :=\frac{2}{\zeta_{2}}\left(a_{4}+a_{5}+a_{6}+L a_{7}\right), \\
k_{3} & :=\frac{2\left(b_{4}+b_{5}+b_{6}+L b_{7}\right)^{2}}{\zeta_{2}^{2}},
\end{aligned}
$$

where all constants come from Assumptions 6 and 9 and $L$ is defined as in Step 1 of this proof. Let $\|u\|_{\mathcal{L}_{2}} \leq \Delta_{L_{u_{1}}},\|\dot{u}\|_{\mathcal{L}_{2}} \leq \Delta_{L_{u_{2}}}$, and $\|w\|_{\mathcal{L}_{2}} \leq \Delta_{L_{w}}$. Consider the set $\Omega_{1}=\left\{(x, \xi, \chi, e) \in \mathbb{R}^{n} \times \mathbb{R}^{m} \times \mathbb{R}^{q} \times \mathbb{R}^{n}||(x, \xi, \chi, e) \mid \leq \bar{\alpha}\left(\Delta_{L}\right)\right\}$ which is a subset of $B_{\rho}=\left\{(x, \xi, \chi, e) \in \mathbb{R}^{n} \times \mathbb{R}^{m} \times \mathbb{R}^{q} \times \mathbb{R}^{n}||(x, \xi, \chi, e) \mid \leq \underline{\alpha}^{-1} \circ \bar{\alpha}\left(\Delta_{L}\right)\right\}$.

Claim: If $\left(x_{0}, \xi_{0}, \chi_{0}, e_{0}\right) \in \Omega_{1}$ for some $t_{0} \geq 0$, then $(x(t), \xi(t), \chi(t), e(t)) \in \Omega_{1}$ for all $t \geq t_{0}$. Proof of claim: We proof our claim by contradiction. Assume there exists $v>0$ and some $t_{1}>t_{0}$ such that

$$
V_{3}\left(t_{1}, e\left(t_{1}\right), x\left(t_{1}\right), \chi\left(t_{1}\right)\right) \geq \bar{\alpha}\left(\Delta_{L}\right)+v .
$$


Let $t_{1}$ be minimal value of $t$ such that the above inequality holds (for a fixed $v$ ). Hence, $V_{3}(t, e(t), x(t), \chi(t))>\bar{\alpha}\left(\Delta_{L}\right)$ for some $t$ close to $t_{1}$. Since $|e| \geq \bar{\alpha}^{-1}\left(V_{3}\right)$ and $V_{3}(t, e(t), x(t), \chi(t))>\bar{\alpha}\left(\Delta_{L}\right)$, we have

$$
|e| \geq \Delta_{L} .
$$

Then, it follows from (A14) that the inequality on the left-hand side of (A13) holds for each $t$ in the neighbourhood of $t_{1}$, and the continuous function $V_{3}(t, e(t), x(t), \chi(t))$ has negative derivative near $t_{1}$. Thus, $V_{3}(t, e(t), x(t), \chi(t))>V_{3}\left(t_{1}, e\left(t_{1}\right), x\left(t_{1}\right), \chi\left(t_{1}\right)\right)$ for some $t \in\left(t_{0}, t_{1}\right)$, contradicting minimality of $t_{1}$. Therefore, $\Omega_{1}$ must indeed be invariant, as claimed. This completes the proof of the claim.

Since $\Omega_{1}$ is an invariant set, we know that any trajectory starting within the set will remain in it, and subsequently, in $B_{\rho}$. This implies that the norm infinity of the estimation error will remain bounded for all $t \geq t_{0}$. Moreover, we know from Lemma 1 and Corollary 2 (see Step 1 of this proof) that the states of the system and the state of the observer have finite and bounded infinity norm for all $\epsilon \in\left(0, \epsilon_{L}^{*}\right),\left|\left(x_{0}, \xi_{0}, \chi_{0}, e_{0}\right)\right| \leq \Delta_{L},\|u\|_{\infty} \leq \Delta_{L_{u_{1}}},\|\dot{u}\|_{\infty} \leq \Delta_{L_{u_{2}}},\|w\|_{\infty} \leq \Delta_{L_{w}}$ and $t \geq t_{0} \geq 0$. Therefore, we can now integrate $(\mathrm{A} 12)$ as follows

$$
\begin{gathered}
V_{3}(t, e(t), x(t), \xi(t))-V_{3}\left(t_{0}, e\left(t_{0}\right), x\left(t_{0}\right), \xi\left(t_{0}\right)\right) \leq-\frac{1}{2} \zeta_{2} \int_{t_{0}}^{t} \alpha_{V_{3}}^{2}(|e(\tau)|) d \tau+\epsilon\left(a_{4}+a_{5}+a_{6}+L a_{7}\right) \int_{t_{0}}^{t} \alpha_{V_{1}}^{2}(|x(\tau)|) d \tau \\
+\epsilon \int_{t_{0}}^{t} \gamma_{5}^{2}(|u(\tau)|) d \tau+\epsilon \int_{t_{0}}^{t} \gamma_{6}^{2}(|u(\tau)|) d \tau+\frac{\bar{k}_{1}}{\zeta_{2}} \int_{t_{0}}^{t} \alpha_{W}^{2}(|\xi(\tau)|) d \tau+\int_{t_{0}}^{t} \gamma_{V_{3}}(|w(\tau)|) d \tau,
\end{gathered}
$$

where $x(t)$ and $\xi(t)$ are the solutions of (6). We use the fact that $V_{3}(t, e(t), x(t), \xi(t)) \geq 0$ to obtain

$$
\begin{aligned}
\frac{1}{2} \zeta_{2} \int_{t_{0}}^{t} \alpha_{V_{3}}^{2}(|e(\tau)|) d \tau & \leq V_{3}\left(t_{0}, e\left(t_{0}\right), x\left(t_{0}\right), \xi\left(t_{0}\right)\right)+\epsilon\left(a_{4}+a_{5}+a_{6}+L a_{7}\right) \int_{t_{0}}^{t} \alpha_{V_{1}}^{2}(|x(\tau)|) d \tau+\epsilon \int_{t_{0}}^{t} \gamma_{5}^{2}(|u(\tau)|) d \tau \\
& +\epsilon \int_{t_{0}}^{t} \gamma_{6}^{2}(|u(\tau)|) d \tau+\frac{\bar{k}_{1}}{\zeta_{2}} \int_{t_{0}}^{t} \alpha_{W}^{2}(|\xi(\tau)|) d \tau+\int_{t_{0}}^{t} \gamma_{V_{3}}(|w(\tau)|) d \tau .
\end{aligned}
$$

It follows from 24 that $V_{3}\left(t_{0}, e\left(t_{0}\right), x\left(t_{0}\right), \xi\left(t_{0}\right)\right) \leq \bar{\alpha}_{V_{3}}\left(\left|e_{0}\right|\right)$. Therefore, it follows from A18p that A2 holds for all $\epsilon \in\left(0, \epsilon_{L}^{*}\right)$, $\left|\left(x_{0}, \xi_{0}, \chi_{0}, e_{0}\right)\right| \leq \Delta_{L}$, for any input satisfying $\|u\|_{\infty} \leq \Delta_{L_{u_{1}}},\|\dot{u}\|_{\infty} \leq \Delta_{L_{u_{2}}},\|u\|_{\mathcal{L}_{2}} \leq \Delta_{L_{u_{1}}},\|\dot{u}\|_{\mathcal{L}_{2}} \leq \Delta_{L_{u_{2}}}$, for any $\|w\|_{\infty} \leq$ $\Delta_{L_{w}},\|w\|_{\mathcal{L}_{2}} \leq \Delta_{L_{w}}$ and for all $t \geq t_{0} \geq 0$, where $k_{i}(i=1,2,3)$ are given by A16. This completes the proof.

Proof of Theorem 1. We split the proof in four steps. In the first step, we prove that (38) holds under Assumptions 1 - 9 Then, we show that $(39)$ holds under the same assumptions. In the third step, we demonstrate that the error dynamics satisfy (40). Finally, we show that the error dynamics also satisfy $(41)$.

Step 1) Let Assumptions 1 - 9 hold. Define the class- $\mathcal{K} \mathcal{L}$ function

$$
\beta_{T_{1}}(r, s):=\beta_{e}\left(2\left[\beta_{e}\left(r, \frac{s}{2}\right)+\gamma_{\xi}\left(2 \beta_{\xi}(r, 0)\right)\right], \frac{s}{2}\right)+\gamma_{\xi}\left(2 \beta_{\xi}\left(r, \frac{s}{2 \epsilon}\right)\right),
$$

where $\beta_{e}(\cdot, \cdot) \in \mathcal{K} \mathcal{L}$ and $\gamma_{\xi}(\cdot) \in \mathcal{K}_{\infty}$ come from Lemma 2 , and $\beta_{\xi}(\cdot, \cdot) \in \mathcal{K} \mathcal{L}$ is given in Corollary 1. Define the class- $\mathcal{K}_{\infty}$ function

$$
\gamma_{T_{1}}(s):=\gamma_{w}(s)+\beta_{e}\left(2 \gamma_{w}(s), 0\right)
$$

where $\gamma_{w}(\cdot) \in \mathcal{K}_{\infty}$ comes from Lemma 2. Let $\Delta>0, \Delta_{u_{1}}>0, \Delta_{u_{2}}>0, \Delta_{w}>0$ and $\mu>0$ be given such that $\left|\left(x_{0}, \xi_{0}, \chi_{0}, e_{0}\right)\right| \leq$ $\Delta,\|u\|_{\infty} \leq \Delta_{u_{1}},\|\dot{u}\|_{\infty} \leq \Delta_{u_{2}}$, and $\|w\|_{\infty} \leq \Delta_{w}$. Define

$$
\mu_{T_{1}}:=\beta_{e}(2 \mu, 0) \text {. }
$$

Let $\left(\bar{\Delta}, \bar{\Delta}_{u_{1}}, \bar{\Delta}_{u_{2}}, \bar{\Delta}_{w}\right)$ be defined as $\bar{\Delta}:=\Delta, \bar{\Delta}_{u_{1}}:=\Delta_{u_{1}}, \bar{\Delta}_{u_{2}}:=\Delta_{u_{2}}, \bar{\Delta}_{w}:=\Delta_{w}$. Using Corollary 2 , let $\left(\bar{\Delta}, \bar{\Delta}_{u_{1}}, \bar{\Delta}_{u_{2}}, \bar{\Delta}_{w}\right)$ generate $\hat{\epsilon}^{*}>0$ and $\Upsilon>0$ such that 32 holds for all $\epsilon \in\left(0, \hat{\epsilon}^{*}\right)$. We now introduce $\Delta_{\chi}:=\alpha_{c_{1}}(\bar{\Delta})+\Upsilon$, where $\alpha_{c_{1}}(\cdot)$ and $\Upsilon>0$ come from 32 in Corollary 2. From the choice of $\left(\bar{\Delta}, \bar{\Delta}_{u_{1}}, \bar{\Delta}_{u_{2}}, \bar{\Delta}_{w}\right)$, we conclude that $|\chi(t)| \leq \Delta_{\chi}$ for all $\left|\left(x_{0}, \xi_{0}, \chi_{0}, e_{0}\right)\right| \leq \bar{\Delta}$, $u \in B_{u_{1}}, \dot{u} \in B_{u_{2}}$, and $t \geq t_{0} \geq 0$ where $B_{u_{1}}=\left\{u \in \mathbb{R}^{r}|| u \mid \leq \Delta_{u_{1}}\right\}$ and $B_{u_{2}}=\left\{\dot{u} \in \mathbb{R}^{r}|| \dot{u} \mid \leq \Delta_{u_{2}}\right\}$. Define $\left(\tilde{\Delta}, \tilde{\Delta}_{u_{1}}, \tilde{\Delta}_{u_{2}}, \tilde{\mu}\right)$ 
as $\tilde{\Delta}:=\Delta, \tilde{\Delta}_{u_{1}}:=\Delta_{u_{1}}, \tilde{\Delta}_{u_{2}}:=\Delta_{u_{2}}$, and

$$
\tilde{\mu}:=\alpha_{W}^{-1}\left(\sqrt{\frac{\zeta_{2}^{2}}{8\left[b_{4}+b_{5}+b_{6}+L b_{7}\right]^{2}} \tilde{\alpha}_{V_{3}} \circ \bar{\alpha}_{V_{3}}^{-1} \circ \underline{\alpha}_{V_{3}}\left(\frac{\mu}{2}\right)}\right),
$$

where all of the above constants come from Assumptions 6 and 9. the class- $\mathcal{K}_{\infty}$ functions come from Assumptions 3 and 6 and $L>0$ is such that $\left|\partial h_{o} / \partial \chi\right| \leq L$ for all $\chi \in B_{1}$ with $B_{1}=\left\{\chi \in \mathbb{R}^{q}|| \chi \mid \leq \Delta_{1}\right\}$ where $\Delta_{1}:=\Delta_{\chi}$. From the choice of $\left(\tilde{\Delta}, \tilde{\Delta}_{u_{1}}, \tilde{\Delta}_{u_{2}}, \tilde{\mu}\right)$, we generate $\bar{\epsilon}^{*}$ such that Corollary 1 hold. Define $\left(\Delta_{L}, \Delta_{L_{u_{1}}}, \Delta_{L_{u_{2}}}, \Delta_{L_{w}}\right)$ as $\Delta_{L}:=\Delta, \Delta_{L_{u_{1}}}:=\Delta_{u_{1}}$, $\Delta_{L_{u_{2}}}:=\Delta_{u_{2}}, \Delta_{L_{w}}:=\Delta_{w}$. Let Lemma 2 hold with the choice of $\left(\Delta_{L}, \Delta_{L_{u_{1}}}, \Delta_{L_{u_{2}}}, \Delta_{L_{w}}\right)$, which means that Lemma 1 holds too. Introduce $\Delta_{x}:=\beta_{L_{1}}(\Delta, 0)+\gamma_{L_{1}}\left(\Delta_{u_{1}}\right)+\tilde{\gamma}_{L_{1}}\left(\Delta_{u_{1}}\right)+\hat{\gamma}_{L_{1}}\left(\Delta_{u_{2}}\right)+\mu_{L_{1}}$ where $\beta_{L_{1}}(\cdot, \cdot), \gamma_{L_{1}}(\cdot), \tilde{\gamma}_{L_{1}}(\cdot), \hat{\gamma}_{L_{1}}(\cdot)$ and $\mu_{L_{1}}$ come from (17) in Lemma 1. We now define an auxiliary constant $\bar{C}:=\epsilon c_{1}+c_{2}$ with

$$
\begin{aligned}
& c_{1}=\left(a_{4}+a_{5}+a_{6}+L a_{7}\right) \alpha_{V_{1}}^{2}\left(\Delta_{x}\right)+\gamma_{5}^{2}\left(\Delta_{u_{1}}\right)+\gamma_{6}^{2}\left(\Delta_{u_{1}}\right), \\
& c_{2}=\frac{\left[b_{4}+b_{5}+b_{6}+L b_{7}\right]^{2}}{\zeta_{2}} \alpha_{W}^{2}(\tilde{\mu}),
\end{aligned}
$$

where all constants come from Assumption 6 and $9 \tilde{\mu}$ is defined as in $\sqrt{\text { A22 }}, L>0$ is as defined above, $\alpha_{V_{1}}(\cdot), \alpha_{W}(\cdot)$, and $\gamma_{i}(\cdot)(i=$ 5,6) come from Assumptions 2, 3 and 9 respectively. Define the auxiliary constant

$$
\hat{C}:=\underline{\alpha}_{V_{3}}^{-1} \circ \bar{\alpha}_{V_{3}} \circ \tilde{\alpha}_{V_{3}}^{-1}\left(\frac{4}{\zeta_{2}} \bar{C}\right),
$$

where it is observed that $\hat{C}$ can be made small by reducing $\bar{C}$, which implies to reduce $\epsilon$ and $\tilde{\mu}$. The weak triangle inequality for comparison functions leads to

$$
\hat{C} \leq \underline{\alpha}_{V_{3}}^{-1} \circ \bar{\alpha}_{V_{3}} \circ \tilde{\alpha}_{V_{3}}^{-1}\left(\frac{8}{\zeta_{2}} \epsilon c_{1}\right)+\underline{\alpha}_{V_{3}}^{-1} \circ \bar{\alpha}_{V_{3}} \circ \tilde{\alpha}_{V_{3}}^{-1}\left(\frac{8}{\zeta_{2}} c_{2}\right) .
$$

Note that $\tilde{\mu}$ in $\mathrm{A} 22$ is such that the second term on the right-hand side of $\mathrm{A} 26$ is half of $\mu$, i.e., $\frac{1}{2} \mu=\underline{\alpha}_{V_{3}}^{-1} \circ \bar{\alpha}_{V_{3}} \circ \tilde{\alpha}_{V_{3}}^{-1}\left(\frac{8}{\zeta_{2}} c_{2}\right)$. So, let $\left(\Delta, \Delta_{u_{1}}, \Delta_{u_{2}}, \mu\right)$ generate

$$
\epsilon_{a}^{*}:=\frac{\zeta_{2}\left[\tilde{\alpha}_{V_{3}}^{-1} \circ \bar{\alpha}_{V_{3}}^{-1} \circ \underline{\alpha}_{V_{3}}\left(\frac{1}{2} \mu\right)\right]}{\left.8\left[\left(a_{4}+a_{5}+a_{6}+L a_{7}\right) \alpha_{V_{1}}^{2}\left(\Delta_{x}\right)+\gamma_{5}^{2}\left(\Delta_{u_{1}}\right)+\gamma_{6}^{2}\left(\Delta_{u_{1}}\right)\right]\right]},
$$

such that $\mu>\hat{C}$ holds for all $\epsilon \in\left(0, \epsilon_{a}^{*}\right)$, which implies that $\frac{1}{2} \mu>\underline{\alpha}_{V_{3}}^{-1} \circ \bar{\alpha}_{V_{3}} \circ \tilde{\alpha}_{V_{3}}^{-1}\left(\frac{8}{\zeta_{2}} \epsilon c_{1}\right)$. Hence, define

$$
\epsilon^{*}:=\min \left\{\epsilon_{L}^{*}, \bar{\epsilon}^{*}, \epsilon_{a}^{*}\right\} .
$$

Note that $\epsilon_{a}^{*}$ is given by $\mathrm{A} 27, \bar{\epsilon}^{*}$ is generated by Corollary 1 , and $\epsilon_{L}^{*}$ in $\mathrm{A} 28$ comes from $\mathrm{A} 6$ in Lemma 2 and implies that Lemma 1 and Corollary 2 hold. It follows from Lemma 2 that

$$
\begin{aligned}
|e(t)| \leq & \beta\left(\left|e_{0}\right|, t-t_{0}\right)+\underline{\alpha}_{V_{3}}^{-1} \circ \bar{\alpha}_{V_{3}} \circ \tilde{\alpha}_{V_{3}}^{-1}\left(\frac { 4 } { \zeta _ { 2 } } \left[\epsilon\left(a_{4}+a_{5}+a_{6}+L a_{7}\right) \alpha_{V_{1}}^{2}\left(\left\|x\left[t_{0}, t\right]\right\|\right)\right.\right. \\
& \left.\left.+\epsilon \gamma_{5}^{2}\left(\| u\left[t_{0}, t\right]||\right)+\epsilon \gamma_{6}^{2}\left(\| u\left[t_{0}, t\right]||\right)+\frac{\bar{k}_{1}}{\zeta_{2}} \alpha_{W}^{2}\left(\left\|\xi\left[t_{0}, t\right]\right\|\right)+\gamma_{V_{3}}\left(\left\|w\left[t_{0}, t\right]\right\|\right)\right]\right),
\end{aligned}
$$

with $\bar{k}_{1}=\left(b_{4}+b_{5}+b_{6}+L b_{7}\right)^{2}$. By virtue of Lemma 1 and Corollary 1 , we have that $x(t)$ and $\xi(t)$ are bounded signal inputs to the error dynamics. We now use the cascade properties of the system to conclude the result. By using the ISS approach for interconnected systems proposed in Lemma 4.7 of Khalil ${ }^{29}$, we have that A29, yield to

$$
\begin{aligned}
|e(t)| & \leq \beta_{T_{1}}\left(\left|\left(x_{0}, \xi_{0}, e_{0}\right)\right|, t-t_{0}\right)+\beta_{e}\left(2 \underline{\alpha}_{V_{3}}^{-1} \circ \bar{\alpha}_{V_{3}} \circ \tilde{\alpha}_{V_{3}}^{-1}\left(\frac{4}{\zeta_{2}}\left(\epsilon c_{1}+c_{2}\right)\right), \frac{t-t_{0}}{2}\right) \\
& +\gamma_{T_{1}}\left(\left\|w\left[t_{0}, t\right]\right\|\right)+\underline{\alpha}_{V_{3}}^{-1} \circ \bar{\alpha}_{V_{3}} \circ \tilde{\alpha}_{V_{3}}^{-1}\left(\frac{8}{\zeta_{2}} \epsilon c_{1}\right)+\underline{\alpha}_{V_{3}}^{-1} \circ \bar{\alpha}_{V_{3}} \circ \tilde{\alpha}_{V_{3}}^{-1}\left(\frac{8}{\zeta_{2}} c_{2}\right),
\end{aligned}
$$

where $\beta_{T_{1}}(\cdot, \cdot) \in \mathcal{K} \mathcal{L}$ and $\gamma_{T_{1}}(\cdot) \in \mathcal{K}_{\infty}$ are given by A19) and A20, respectively. Therefore, by the fact that $\mu>\hat{C}$, $\beta(r, 0) \geq \beta(r, s)$, and using $(\mathrm{A} 21)$, we conclude that 38$\rangle$ holds for all $\epsilon \in\left(0, \epsilon^{*}\right)$ and for all $\left|\left(x_{0}, \xi_{0}, \chi_{0}, e_{0}\right)\right| \leq \Delta,\|u\|_{\infty} \leq \Delta_{u_{1}}$, $\|\dot{u}\|_{\infty} \leq \Delta_{u_{2}},\|w\|_{\infty} \leq \Delta_{w}$ and $t \geq t_{0}>0$. 
Step 2) We now prove that 39 holds under Assumptions 1 - 9 Define the functions $\bar{\beta}_{T_{1}}(\cdot, \cdot) \in \mathcal{K} \mathcal{L}$ and $\bar{\gamma}_{T_{1}}(\cdot) \mathcal{K}_{\infty}$

$$
\begin{aligned}
\bar{\beta}_{T_{1}}(r, s) & :=\beta_{e}(r, s), \\
\gamma_{T_{1}}(s) & :=\gamma_{w}(s),
\end{aligned}
$$

where $\beta_{e}(\cdot, \cdot) \in \mathcal{K} \mathcal{L}$ and $\gamma_{w}(\cdot) \in \mathcal{K}_{\infty}$ come from Lemma 2. For the given $\Delta>0, \Delta_{u_{1}}>0, \Delta_{u_{2}}>0, \Delta_{w}>0$ and $\mu>0$, let $\epsilon^{*}>0$ be as defined in A28. As showed in Step 1, Lemmas 1 and 2 and Corollaries 1 and 2 hold. Using Corollary 1 , let $\left(\tilde{\Delta}, \tilde{\Delta}_{u_{1}}, \tilde{\Delta}_{u_{2}}, \tilde{\mu}\right)$ generate $T^{*}>0$ such that 20 in Corollary 1 holds for all $\epsilon \in\left(0, \bar{\epsilon}^{*}\right),\left|\left(x_{0}, \xi_{0}\right)\right| \leq \tilde{\Delta},\|u\|_{\infty} \leq \tilde{\Delta}_{u_{1}},\|\dot{u}\|_{\infty} \leq \tilde{\Delta}_{u_{2}}, t \geq \epsilon T^{*}+t_{0}$. Hence, $T^{*}$ is given by

$$
T^{*}:=\epsilon \frac{v-v}{K}
$$

where $v:=\bar{\alpha}_{W} \circ \tilde{\alpha}_{W}^{-1}\left(\epsilon \frac{4}{\zeta_{3}}\left[\left(b_{2}+b_{3}\right)^{2} \alpha_{V_{1}}^{2}\left(\tilde{\Delta}_{x}\right)+\gamma_{2}^{2}\left(\tilde{\Delta}_{u_{1}}\right)+\gamma_{3}^{2}\left(\tilde{\Delta}_{u_{1}}\right)+\gamma_{4}^{2}\left(\tilde{\Delta}_{u_{2}}\right)\right]\right)$ with $\tilde{\Delta}_{x}=\Delta_{x}, \tilde{\alpha}_{W}(\cdot):=\alpha_{W}^{2}(\cdot), v:=\bar{\alpha}_{W}(\tilde{\Delta})$, $K=\min \left\{\hat{\alpha}_{W}(|\xi|)\right\}$ over the set $\left\{\tilde{\Delta} \leq|\xi| \leq \tilde{\Delta}_{\xi}\right\}$ with $\hat{\alpha}_{W}(\cdot)=\frac{\zeta_{3}}{4} \alpha_{W}^{2} \circ \bar{\alpha}_{W}^{-1}(\cdot)$ and $\tilde{\Delta}_{\xi}=\underline{\alpha}_{W}^{-1} \circ \bar{\alpha}_{W}(\tilde{\Delta})$, where all the constants and class- $\mathcal{K}_{\infty}$ functions come from Assumptions 3, 4 and 5.

To show the result, we consider $\mathrm{A} 29$ which comes from Lemma 2. It is observed that $x(t)$ and $\xi(t)$ are essentially bounded signal inputs to the error dynamics. We know from the SPA result in Corollary 1 that the fast state rapidly converges, and it becomes ultimately bounded by $\tilde{\mu}$ after a finite time $T^{*}>0$ defined by $(\mathrm{A} 33)$. This occurs because $\beta_{\xi}(\cdot, \cdot) \in \mathcal{K} \mathcal{L}$ in $(19)$ quickly converges to zero. Hence, $\left\|\xi\left[\epsilon T^{*}, t\right]\right\| \leq \tilde{\mu}$ for all $t \geq \epsilon T^{*}+t_{0}$ where $\tilde{\mu}$ is given by (A22). Moreover, $\left\|x\left[t_{0}, t\right]\right\| \leq \Delta_{x}$, and $\left\|u\left[t_{0}, t\right]\right\| \leq \Delta_{u_{1}}$. Therefore, by considering $c_{1}$ and $c_{2}$ in (A23) and (A24), (A29) leads to

$$
|e(t)| \leq \bar{\beta}_{T_{1}}\left(\left|e\left(t_{0}\right)\right|, t-t_{0}\right)+\underline{\alpha}_{V_{3}}^{-1} \circ \bar{\alpha}_{V_{3}} \circ \tilde{\alpha}_{V_{3}}^{-1}\left(\frac{8}{\zeta_{2}} \epsilon c_{1}\right)+\underline{\alpha}_{V_{3}}^{-1} \circ \bar{\alpha}_{V_{3}} \circ \tilde{\alpha}_{V_{3}}^{-1}\left(\frac{8}{\zeta_{2}} c_{2}\right)+\bar{\gamma}_{T_{1}}\left(\left\|w\left[t_{0}, t\right]\right\|\right),
$$

for all $\epsilon \in\left(0, \epsilon^{*}\right)$ and for all $t \geq \epsilon T^{*}+t_{0}$, where $\bar{\beta}_{T_{1}}(\cdot, \cdot) \in \mathcal{K}_{\infty}$ and $\bar{\gamma}_{T_{1}}(\cdot) \in \mathcal{K}_{\infty}$ given by $\mathrm{A} 31$ and $\mathrm{A} 32$. Note that the sum of second and third terms on the right-hand side of A34 is equal to right hand side of A26, which is smaller than $\mu$ for all $\epsilon \in\left(0, \epsilon^{*}\right)$. Therefore, we conclude that $\left[39\right.$ holds for all $\epsilon \in\left(0, \epsilon^{*}\right),\left|\left(x_{0}, \xi_{0}, \chi_{0}, e_{0}\right)\right| \leq \Delta,\|u\|_{\infty} \leq \Delta_{u_{1}},\|\dot{u}\|_{\infty} \leq \Delta_{u_{2}}$, $\|w\|_{\infty} \leq \Delta_{w}$ and $t \geq \epsilon T^{*}+t_{0}$.

Step 3) We now prove the $\mathcal{L}_{2}$ stability property 40 . Define

$$
k_{T_{1}}:=\frac{2}{\zeta_{2}},
$$

and the class- $\mathcal{K}_{\infty}$ functions

$$
\begin{aligned}
& \alpha_{T_{1}}(s):=k_{T_{1}} \bar{\alpha}_{V_{3}}(s), \\
& \bar{\alpha}_{T_{1}}(s):=\frac{k_{T_{1}}}{\zeta_{2}}\left(b_{4}+b_{5}+b_{6}+L b_{7}\right)^{2} \alpha_{W_{c}}(s),
\end{aligned}
$$

where $\alpha_{W_{c}} \in \mathcal{K}_{\infty}$ come from Corollary 1. For the given $\Delta>0, \Delta_{u_{1}}>0, \Delta_{u_{2}}>0, \Delta_{w}>0$ and $\mu>0$, let $\left|\left(x_{0}, \xi_{0}, \chi_{0}, e_{0}\right)\right| \leq \Delta$, $\left.\left\|\left.u\right|_{\mathcal{L}_{2}} \leq \Delta_{u_{1}},\right\| \dot{u}\left\|_{\mathcal{L}_{2}} \leq \Delta_{u_{2}},\right\| w\right|_{\mathcal{L}_{2}} \leq \Delta_{w}$. Let Lemmas 1 and 2 and Corollary 2 hold as in Step 1 of this proof where the $\mathcal{L}_{2}$ results hold for any input satisfying $\|u\|_{\infty} \leq \Delta_{u_{1}},\|\dot{u}\|_{\infty} \leq \Delta_{u_{2}},\|u\|_{\mathcal{L}_{2}} \leq \Delta_{u_{1}},\|\dot{u}\|_{\mathcal{L}_{2}} \leq \Delta_{u_{2}}$, and for any $\|w\|_{\infty} \leq \Delta_{w}$, $\|w\|_{\mathcal{L}_{2}} \leq \Delta_{w}$. Define $\left(\tilde{\Delta}, \tilde{\Delta}_{u_{1}}, \Delta_{u_{2}}, \tilde{\mu}\right)$ as $\tilde{\Delta}:=\Delta, \tilde{\Delta}_{u_{1}}=\Delta_{u_{1}}, \tilde{\Delta}_{u_{2}}=\Delta_{u_{2}}$ and

$$
\tilde{\mu}:=\alpha_{W}^{-1}\left(\sqrt{\frac{\zeta_{2}^{2}}{4\left(b_{4}+b_{5}+b_{6}+L b_{7}\right)^{2}} \mu}\right) .
$$

From the choice of $\left(\tilde{\Delta}, \tilde{\Delta}_{u_{1}}, \Delta_{u_{2}}, \tilde{\mu}\right)$, we generate $\bar{\epsilon}^{*}>0$ such that Corollary 1 holds. Define

$$
\mu_{\mathcal{L}_{2}}:=\frac{k_{T_{1}}}{\zeta_{2}}\left(b_{4}+b_{5}+b_{6}+L b_{7}\right)^{2} \alpha_{W}^{-1}\left(\sqrt{\frac{\zeta_{2}^{2}}{4\left(b_{4}+b_{5}+b_{6}+L b_{7}\right)^{2}} \mu}\right),
$$


where $\alpha_{w}(\cdot) \in \mathcal{K}_{\infty}$ and the rest of the constant come from Assumptions 34 and 9 and $L$ is defined as in Step 1 of this proof. Let $\epsilon_{b}^{*}>0$ be such that $\epsilon \frac{2}{\zeta_{2}}\left[\left(a_{4}+a_{5}+a_{6}+L a_{7}\right) \alpha_{V_{1}}^{2}\left(\Delta_{x}\right)+\gamma_{5}^{2}\left(\Delta_{u_{1}}\right)+\gamma_{6}^{2}\left(\Delta_{u_{1}}\right)\right] \leq \frac{1}{2} \mu$ for all $\epsilon \in\left(0, \epsilon_{b}^{*}\right)$. Hence, define

$$
\epsilon_{b}^{*}:=\frac{\zeta_{2} \mu}{4\left[\left(a_{4}+a_{5}+a_{6}+L a_{7}\right) \alpha_{V_{1}}^{2}\left(\Delta_{x}\right)+\gamma_{5}^{2}\left(\Delta_{u_{1}}\right)+\gamma_{6}^{2}\left(\Delta_{u_{2}}\right)\right]},
$$

and

$$
\epsilon_{\mathcal{L}_{2}}^{*}:=\min \left\{\epsilon_{L}^{*}, \bar{\epsilon}^{*}, \epsilon_{b}^{*}\right\},
$$

where $\epsilon_{L}^{*}$ and $\bar{\epsilon}^{*}$ come from Lemma 2 and Corollary 1, respectively. We now consider (A2) in Lemma 2 and 21 in Corollary 1. Note that $(\mathrm{A} 2)$ holds for all $\epsilon \in\left(0, \epsilon^{*}\right)$ since $\epsilon_{L}^{*} \leq \epsilon^{*}$. It follows that (A2) can be written as follows

$$
\begin{aligned}
\int_{t_{0}}^{t} \alpha_{V_{3}}^{2}(|e(\tau)|) d \tau & \leq \frac{2}{\zeta_{2}} \bar{\alpha}_{V_{3}}\left(\left|e_{0}\right|\right)+\epsilon \frac{2}{\zeta_{2}}\left(a_{4}+a_{5}+a_{6}+L a_{7}\right) \int_{t_{0}}^{t} \alpha_{V_{1}}^{2}(|x(\tau)|) d \tau+\epsilon \frac{2}{\zeta_{2}} \int_{t_{0}}^{t} \gamma_{5}^{2}(|u(\tau)|) d \tau \\
& +\epsilon \frac{2}{\zeta_{2}} \int_{t_{0}}^{t} \gamma_{6}^{2}(|u(\tau)|) d \tau+\frac{2 \bar{k}_{1}}{\zeta_{2}^{2}}\left(\epsilon \alpha_{W_{c}}\left(\left|\xi_{0}\right|\right)+\tilde{\mu}\left(t-t_{0}\right)\right)+\frac{2}{\zeta_{2}} \int_{t_{0}}^{t} \gamma_{V_{3}}(|w(\tau)|) d \tau,
\end{aligned}
$$

where $\alpha_{W_{c}}(\cdot)$ comes from Corollary 1 and $\bar{k}_{1}=\left(b_{4}+b_{5}+b_{6}+L b_{7}\right)^{2}$. We use the fact that $|x(t)| \leq \Delta_{x}$ and $|u(t)| \leq \Delta_{u_{1}}$ to obtain

$$
\begin{aligned}
\int_{t_{0}}^{t} \alpha_{V_{3}}^{2}(|e(\tau)|) d \tau & \leq \frac{2}{\zeta_{2}} \bar{\alpha}_{V_{3}}\left(\left|e_{0}\right|\right)+\epsilon \frac{2}{\zeta_{2}}\left[\left(a_{4}+a_{5}+a_{6}+L a_{7}\right) \alpha_{V_{1}}^{2}\left(\Delta_{x}\right)+\gamma_{5}^{2}\left(\Delta_{u_{1}}\right)+\gamma_{6}^{2}\left(\Delta_{u_{1}}\right)\right] \int_{t_{0}}^{t} d \tau \\
& +\frac{2 \bar{k}_{1}}{\zeta_{2}^{2}}\left(\epsilon \alpha_{W_{c}}\left(\left|\xi_{0}\right|\right)+\tilde{\mu}\left(t-t_{0}\right)\right)+\frac{2}{\zeta_{2}} \int_{t_{0}}^{t} \gamma_{V_{3}}(|w(\tau)|) d \tau .
\end{aligned}
$$

Note that A43 and $\epsilon \frac{2}{\zeta_{2}}\left[\left(a_{4}+a_{5}+a_{6}+L a_{7}\right) \alpha_{V_{1}}^{2}\left(\Delta_{x}\right)+\gamma_{5}^{2}\left(\Delta_{u_{1}}\right)+\gamma_{6}^{2}\left(\Delta_{u_{1}}\right)\right] \leq \frac{1}{2} \mu$ lead to

$$
\int_{t_{0}}^{t} \alpha_{V_{3}}^{2}(|e(\tau)|) d \tau \leq \frac{2}{\zeta_{2}} \bar{\alpha}_{V_{3}}\left(\left|e_{0}\right|\right)+\epsilon \frac{2 \bar{k}_{1}}{\zeta_{2}^{2}} \alpha_{W_{c}}\left(\left|\xi_{0}\right|\right)+\frac{2}{\zeta_{2}} \int_{t_{0}}^{t} \gamma_{V_{3}}(|w(\tau)|) d \tau+\frac{2 \bar{k}_{1}}{\zeta_{2}^{2}} \tilde{\mu}\left(t-t_{0}\right)+\mu\left(t-t_{0}\right),
$$

for all $\epsilon \in\left(0, \epsilon_{\mathcal{L}_{2}}^{*}\right)$. By using $\alpha_{T_{1}}(\cdot), \bar{\alpha}_{T_{1}}(\cdot) \in \mathcal{K}_{\infty}, k_{T_{1}}$ and $\mu_{\mathcal{L}_{2}}$ given in $\overline{\mathrm{A} 35}-(\mathrm{A} 39)$ and by the fact that $\left|e_{0}\right| \leq\left|\left(x_{0}, \xi_{0}, e_{0}\right)\right|$ and $\left|\xi_{0}\right| \leq\left|\left(x_{0}, \xi_{0}, e_{0}\right)\right|$, we conclude from $(\mathrm{A} 44)$ that $(40)$ holds for all $\epsilon \in\left(0, \epsilon_{\mathcal{L}_{2}}^{*}\right),\left|\left(x_{0}, \xi_{0}, \chi_{0}, e_{0}\right)\right| \leq \Delta$, for any input satisfying $\|u\|_{\infty} \leq \Delta_{u_{1}},\|\dot{u}\|_{\infty} \leq \Delta_{u_{2}},\|u\|_{\mathcal{L}_{2}} \leq \Delta_{u_{1}},\|u\|_{\mathcal{L}_{2}} \leq \Delta_{u_{2}}$, for any $\|w\|_{\infty} \leq \Delta_{w},\|w\|_{\mathcal{L}_{2}} \leq \Delta_{w}$, and for all $t \geq t_{0} \geq 0$.

Step 4) Finally, we demonstrate that the $\mathcal{L}_{2}$ stability property (41). To prove this final result, let Lemmas 1 and 2 and Corollary 2 hold as in previous steps, and let Corollary 1 holds as stated in Step 3 of this proof. Let $k_{T_{1}}>0$ and $\alpha_{T_{1}}(\cdot) \in \mathcal{K}_{\infty}$ be such as defined in $\mathrm{A} 35$ and $\mathrm{A} 36$, respectively. Moreover, define $\epsilon_{\mathcal{L}_{2}}^{*}>0$ as in $\mathrm{A} 41$. . From the choice of $\left(\tilde{\Delta}, \tilde{\Delta}_{u_{1}}, \Delta_{u_{2}}, \tilde{\mu}\right)$ in Step 3 where $\tilde{\mu}$ is given by $(A 38)$, we generate $T^{*}>0$ such that $|\xi(t)|^{2} \leq \tilde{\mu}$ for all $t \geq \epsilon T^{*}+t_{0}$. Since we work with the intersection of the infinity norm and the $\mathcal{L}_{2}$ norm, we use the fact that $|\xi(t)| \leq \tilde{\mu}$ for all $t \geq \epsilon T^{*}+t_{0}$ and we consider $|x(t)| \leq \Delta_{x},|u(t)| \leq \Delta_{u_{1}}$ and $|\dot{u}(t)| \leq \Delta_{u_{2}}$. Therefore, we obtain from $(\mathrm{A} 2)$ that the following holds

$$
\begin{aligned}
\int_{t_{0}}^{t} \alpha_{V_{3}}^{2}(|e(\tau)|) d \tau & \leq \frac{2}{\zeta_{2}} \bar{\alpha}_{V_{3}}\left(\left|e_{0}\right|\right)+\epsilon \frac{2}{\zeta_{2}}\left[\left(a_{4}+a_{5}+a_{6}+L a_{7}\right) \alpha_{V_{1}}^{2}\left(\Delta_{x}\right)+\left[\gamma_{5}^{2}\left(\Delta_{u_{1}}\right)+\gamma_{6}^{2}\left(\Delta_{u_{1}}\right)\right]\right] \int_{t_{0}}^{t} d \tau \\
& +\frac{2 \bar{k}_{1}}{\zeta_{2}^{2}} \alpha_{W}^{2}(\tilde{\mu}) \int_{t_{0}}^{t} d \tau+\frac{2}{\zeta_{2}} \int_{t_{0}}^{t} \gamma_{V_{3}}(|w(\tau)|) d \tau,
\end{aligned}
$$

for all $t \geq \epsilon T^{*}+t_{0}$. From the choice of $\tilde{\mu}$ in $A 38$, we have that $\frac{2 \bar{k}_{1}}{\xi_{2}^{2}} \alpha_{W}^{2}(\tilde{\mu}) \leq \frac{1}{2} \mu$. Moreover, it follows from Step 3 of this proof that

$$
\epsilon \frac{2}{\zeta_{2}}\left[\left(a_{4}+a_{5}+a_{6}+L a_{7}\right) \alpha_{V_{1}}^{2}\left(\Delta_{x}\right)+\left[\gamma_{5}^{2}\left(\Delta_{u_{1}}\right)+\gamma_{6}^{2}\left(\Delta_{u_{1}}\right)\right]\right] \leq \frac{1}{2} \mu,
$$


for all $\epsilon \in\left(0, \epsilon_{b}^{*}\right)$ where $\epsilon_{b}^{*} \leq \epsilon_{\mathcal{L}_{2}}^{*}$. Hence, $\mathrm{A} 45$ leads to

$$
\int_{t_{0}}^{t} \alpha_{V_{3}}^{2}(|e(\tau)|) d \tau \leq \frac{2}{\zeta_{2}} \bar{\alpha}_{V_{3}}\left(\left|e_{0}\right|\right)+\frac{2}{\zeta_{2}} \int_{t_{0}}^{t} \gamma_{V_{3}}(|w(\tau)|) d \tau+\mu\left(t-t_{0}\right) .
$$

Therefore, by using $k_{T_{1}}>0$ as in $(\mathrm{A} 35)$ and $\alpha_{T_{1}}(\cdot) \in \mathcal{K}_{\infty}$ as in $(\mathrm{A} 36)$, it follows from $(\mathrm{A} 47)$ that 41 holds for all $\epsilon \in\left(0, \epsilon_{\mathcal{L}_{2}}^{*}\right)$, $\left|\left(x_{0}, \xi_{0}, \chi_{0}, e_{0}\right)\right| \leq \Delta$, for any input satisfying $\|u\|_{\infty} \leq \Delta_{u_{1}},\|\dot{u}\|_{\infty} \leq \Delta_{u_{2}},\|u\|_{\mathcal{L}_{2}} \leq \Delta_{u_{1}},\|\dot{u}\|_{\mathcal{L}_{2}} \leq \Delta_{u_{2}}$, for any $\left.\|w\|\right|_{\infty} \leq \Delta_{w}$, $\|w\|_{\mathcal{L}_{2}} \leq \Delta_{w}$, and for all $t \geq \epsilon T^{*} t_{0}$. This completes the proof.

\section{References}

1. Kazantzis N, Huynh N, Wright RA. Nonlinear observer design for the slow states of a singularly perturbed system.Computers and Chemical Engineering. 2005;29(4):797-806.

2. Arcak M. Unmodeled Dynamics in Robust Nonlinear Control. Ph.D. Dissertation, Department of Electrical and Computer Engineering, University of California, Santa Barbara; 2000.

3. Fan X, Arcak M. Nonlinear Observer Design for Systems with Multivariable Monotone Nonlinearities. Proceedings of the 41st IEEE Conference on Decision and Control. 2002;1:684-688.

4. Chong MS, Postoyan R, Nešić D, Kuhlmann L, Varsavsky A. A robust circle criterion observer with application to neural mass models. Automatica. 2012;48(11):2986-2989.

5. Johansson A, Medvedev A. An observer for systems with nonlinear output map. Automatica. 2003;39:909-918.

6. Saha D, Valasek J. Observer-Based Sequential Control of a Nonlinear Two-Time-Scale System with Multiple Slow and Fast States. 10th IFAC Symposium on Nonlinear Control and Systems. 2016;49(18):696-701.

7. Saha D, Valasek, J. Observer-Based Sequential Control of a Nonlinear Two-Time-Scale Spring-Mass-Damper System. AIAA Guidance Navigation and Control Conference. 2016.

8. Astolfi D, Marconi L. A High-Gain Nonlinear Observer with Limited Gain Power. IEEE Transactions on Automatic Control. 2015;60(11):3059-3064.

9. G. Besançon, Nonlinear Observers and Applications. Berlin Heidelberg: Springer-Verlag, 2007.

10. H. Khalil, High-Gain Observers in Nonlinear Feedback Control, 1st ed. Society of Industrial and Applied Mathematics, 2017.

11. Kimball JW, Krein PT. Singular Perturbation Theory for DC-DC Converters and Application to PFC Converters. IEEE Transactions on Power Electronics. 2008;23:2970-2981.

12. Kumar A, Christofides PD, Daoutidisia P. Singular perturbation modelling of nonlinear processes with nonexplicit timescale multiplicity. Chemical Engineering Science. 1998;53(8):1491-1504.

13. Upadhyay D, Nieuwstadt M. Modeling of a urea SCR catalyst with automotive applications. Proceedings of ASME International Mechanical Engineering Congress \& Exposition. 2002.

14. Sharma R, Nešić D, Manzie C. Model reduction of a turbocharged (TC) spark ignition (SI) engines. IEEE Transactions on Control and Systems Technology. 2011;19(2):297-310.

15. Kokotović PV, Chow JH, Khalil HK. Singularly Perturbed Systems. Wiley Encyclopaedia of Electrical and Electronics Engineering. 1999.

16. Z. Gajic and M. Lim, "A new filtering method for linear singularly perturbed systems," IEEE Transactions on Automatic Control, vol. 39, no. 9, pp. 1952-1955, 1994. 
17. K. J. Lin, "Composite observer-based feedback design for singularly perturbed systems via LMI approach," in Proceedings of SICE Annual Conference 2010, 2010, pp. 3056-3061.

18. H. Yoo and Z. Gajic, "New designs of reduced-order observer-based controllers controllers for singularly perturbed linear systems," Mathematical Problems Engineering, pp. 1-14, 2017.

19. H. Yoo and Z. Gajic, "New designs of linear observers and observer-based controllers for singularly perturbed linear systems," IEEE Transactions on Automatic Control, vol. 63, no. 11, pp. 3904-3911, 2018.

20. Y. Wang and W. Liu, "Robust observer-based feedback control for Lipschitz singularly perturbed systems," Mathematical Problems in Engineering, 2015.

21. N. Daroogheh, N. Meskin, and K. Khorasani, "Robust hybrid EKF approach for state estimation in multi-scale nonlinear singularly perturbed systems," in Proceedings of IEEE Conference on Decision and Control, 2014, pp. 1047-1054.

22. Cuevas L, Nešić D, Manzie C. Convergence of full-order observers for the slow states of a singularly perturbed system (Part I: Theory). 2018 Australian and New Zealand Control Conference (ANZCC). Melbourne, VIC, 2018, to be published.

23. Cuevas L, Nešić D, Manzie C. Convergence of full-order observers for the slow states of a singularly perturbed system (Part II: Applications). 2018 Australian and New Zealand Control Conference (ANZCC). Melbourne, VIC, 2018, to be published.

24. Cuevas L, Nešić D, Manzie C. Global stability of the error dynamics of an observer designed for the slow states of a singularly perturbed system. The 15th International Conference on Control, Automation, Robotics and Vision. Singapore, 2018 , to be published.

25. Nešić D, Dower P. A Note on Input-to-State Stability and Averaging of Systems with Inputs. IEEE Transactions on Automatic Control. 2001;46(11):1760-1765.

26. Sontag ED. Smooth Stabilization Implies Coprime Factorization. IEEE Transactions on Automatic Control. 1989;34(4):435-443.

27. Sontag ED, Wang, T. On Characterizations of Input-to-State Stability with Respect to Compact Sets. In Nonlinear Control Systems Design 1995 (1st ed.), Elsevier Science: California; 1995.

28. Christofides PD, Teel AR (1996). Singular Perturbations and Input-to-State Stability. IEEE Transactions on Automatic Control. 1996;41(11):1645-1650.

29. Khalil HK. Nonlinear Systems (3rd ed.). New Jersey: Prentice Hall; 2001.

30. Bruno AD. Singular Perturbation in Hamiltonian Mechanics. Hamiltonian Mechanics: Integrability and Chaotic Behavior. 1994;43-49.

31. Georgiou IT. On the Global Geometric Structure of the Dynamics of the Elastic Pendulum. Nonlinear Dynamics. 1999;18(1):51-68.

32. L. Nie and Z. Teng, "Singular perturbation method for global stability of ratio-dependent predator-prey models with stage structure for the prey," Electronic Journal of Differential Equations, vol. 2013, no. 86, pp. 1-9, 2013.

33. Kokotović PV, Khalil HK, O'Reilly J. Singular Perturbation Methods in Control: Analysis and Design, Philadelphia: SIAM; 1999.

34. Saberi A, Khalil HK. Quadratic-type Lyapunov functions for singularly perturbed systems. IEEE Transactions on Automatic Control. 1984;29(6):542-550. 


\section{University Library}

\section{- M M I N E R VA A gateway to Melbourne's research publications}

Minerva Access is the Institutional Repository of The University of Melbourne

Author/s:

Cuevas, L;Neši\#, D;Manzie, C

Title:

Robustness analysis of nonlinear observers for the slow variables of singularly perturbed systems

Date:

2020-09-25

\section{Citation:}

Cuevas, L., Neši\#, D. \& Manzie, C. (2020). Robustness analysis of nonlinear observers for the slow variables of singularly perturbed systems. International Journal of Robust and Nonlinear Control, 30 (14), pp.5628-5656. https://doi.org/10.1002/rnc.5100.

Persistent Link:

http://hdl.handle.net/11343/251881 